\title{
Molecular characterization of Chlamydomonas reinhardtii telomeres and telomerase mutants
}

\author{
Stephan Eberhard ${ }^{1} \mathbb{0}$, Sona Valuchova ${ }^{3}$, Julie Ravat ${ }^{1}$, Jaroslav Fulneček ${ }^{3}$, Pascale Jolivet ${ }^{2}$, Sandrine Bujaldon ${ }^{1}$,

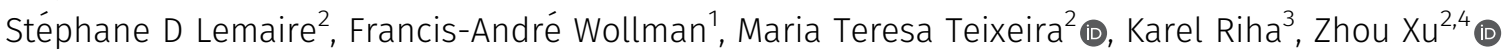

\begin{abstract}
Telomeres are repeated sequences found at the end of the linear chromosomes of most eukaryotes and are required for chromosome integrity. Expression of the reverse-transcriptase telomerase allows for extension of telomeric repeats to counteract natural telomere shortening. Although Chlamydomonas reinhardtii, a photosynthetic unicellular green alga, is widely used as a model organism in photosynthesis and flagella research, and for biotechnological applications, the biology of its telomeres has not been investigated in depth. Here, we show that the $C$. reinhardtii (TTTTAGGG) ${ }_{n}$ telomeric repeats are mostly nondegenerate and that the telomeres form a protective structure, with a subset ending with a $3^{\prime}$ overhang and another subset presenting a blunt end. Although telomere size and length distributions are stable under various standard growth conditions, they vary substantially between 12 genetically close reference strains. Finally, we identify CrTERT, the gene encoding the catalytic subunit of telomerase and show that telomeres shorten progressively in mutants of this gene. Telomerase mutants eventually enter replicative senescence, demonstrating that telomerase is required for long-term maintenance of telomeres in C. reinhardtii.
\end{abstract}

DOI 10.26508/lsa.201900315 | Received 23 January 2019 | Revised 27 May 2019 | Accepted 27 May 2019 | Published online 3 June 2019

\section{Introduction}

Photosynthetic algae are in the highlight of basic and applied research, not only because of their core role for Earth's biosphere in oxygen evolution and carbon fixation but also because of their increased use in biotechnology for the production of proteins, bulk chemicals, and high-value molecules (Scaife et al, 2015; Scranton et al, 2015). Thus, a detailed understanding of algal physiology, including their cell cycle, cell growth, and genome integrity, is of critical importance. Chlamydomonas reinhardtii, also referred to as the "photosynthetic yeast" (Rochaix, 1995), is the most prominent model organism in the green algae lineage. It is widely used for biotechnological applications as well as to study fundamental processes, such as photosynthesis and cilia structure and function (Harris, 2001; Sasso et al, 2018). It has a fully sequenced nuclear genome of $111 \mathrm{Mb}$ distributed over 17 chromosomes (Merchant et al, 2007) and is amenable to powerful genetic approaches (Harris, 2009). Although genetic transformation is available in this organism, targeted nuclear genome modification is still not straightforward, but the emerging CRISPR/Cas9 technology might help solve this limitation (Findinier et al, 2019).

In eukaryotes, telomeres are repeated sequences found at the extremities of linear chromosomes. They are important for chromosome integrity and may limit cell proliferation capacity in some organisms. By progressively shortening with each cell cycle because of the end replication problem, telomeres eventually become too short and trigger a cell cycle arrest termed replicative senescence (Lundblad \& Szostak, 1989; Harley et al, 1990). Most unicellular eukaryotes and germ, stem, and cancer cells in multicellular organisms counteract telomere shortening by expressing telomerase, an enzyme that adds de novo telomere sequences and allows for an unlimited proliferation potential (Pfeiffer \& Lingner, 2013; Wu et al, 2017). Despite the crucial functions of telomeres and telomerase in maintaining genome stability and controlling cell proliferation in many model organisms, including plants, ciliates, fungi, and mammals (Fulcher et al, 2014), telomere biology in algae remains to be investigated in depth.

To our knowledge, only a handful of studies on C. reinhardtii telomeres have been published. Early studies published in the $90 \mathrm{~s}$ showed that (i) C. reinhardtii telomeres are composed of TTTTAGGG repeats, which are different from the Arabidopsis-type TTTAGGG sequence (Petracek et al, 1990); (ii) the size of cloned telomeric repeats ranges from 300 to 600 bp (Petracek et al, 1990; Hails et al, 1995); (iii) they form G-quadruplex structures in vitro (Petracek \& Berman, 1992); and (iv) the Gbp1 protein binds in vitro to singlestranded telomere sequences through two RNA recognition motifs, with a preference for RNA when Gbp1 is monomeric and for DNA when it is dimeric (Petracek et al, 1994; Johnston et al, 1999). More

\footnotetext{
${ }^{1}$ Sorbonne Université, CNRS, UMR 7141, Institut de Biologie Physico-Chimique, Biologie du Chloroplaste et Perception de la Lumière chez les Micro-algues, Paris, France ${ }^{2}$ Sorbonne Université, PSL Research University, CNRS, UMR 8226, Institut de Biologie Physico-Chimique, Laboratoire de Biologie Moléculaire et Cellulaire des Eucaryotes, Paris, France ${ }^{3}$ Central European Institute of Technology, Masaryk University, Brno, Czech Republic ${ }^{4}$ Sorbonne Université, CNRS, UMR 7238, Institut de Biologie ParisSeine, Laboratory of Computational and Quantitative Biology, Paris, France
} 
recently, bioinformatics studies focused on the evolutionary relationships of telomere sequences in green algae (Fulnečková et al, 2015, 2012). Finally, a broad study of telomerase activity in green algae revealed that telomerase activity in C. reinhardtii extracts is low or not detectable (Fulnečková et al, 2013).

To gain a better understanding of $C$. reinhardtii telomere structure and maintenance, we investigated telomere sequence and end structure, analyzed telomere length distribution across different reference strains, identified CrTERT, the gene encoding the catalytic subunit of telomerase, and provided a genetic analysis of telomerase function, thus opening new avenues of research on telomere dynamics, proliferation potential, and genome integrity in c. reinhardtii.

\section{Results}

\section{C. reinhardtii telomeric repeats are mostly nondegenerate with few low-frequency variants}

In their seminal article, Petracek et al (1990) cloned and sequenced a limited number of $C$. reinhardtii telomeric repeats, revealing their canonical TTTTAGGG sequence (Petracek et al, 1990). Telomeric repeats are also identifiable in 18 of 34 chromosome ends on the available v5.5 genome sequence of $C$. reinhardtii (https:// phytozome.jgi.doe.gov; Fig S1A). As the sequenced genome shows some telomeric repeat variations, we analyzed telomeric repeat sequences on a larger scale and looked for putative variants of the canonical telomere sequence. We amplified telomeres by a PCRbased method (Forstemann et al, 2000) using a forward primer specific to a conserved subtelomere-telomere junction common to 10 telomeres from eight different chromosomes (Fig S1A and B). The reverse primer was universal and annealed to a sequence of $c y-$ tosines artificially added at the $3^{\prime}$ end of the telomeres by terminal transferase reaction. After cloning into a plasmid and sequencing, we analyzed 32 telomere sequences, encompassing 709 repeats. We found that $\sim 90 \%(n=636)$ of the repeats corresponded to the canonical sequence TTTTAGGG. We also detected variants such as TTTAGGG (corresponding to the canonical Arabidopsis thaliana sequence, $\mathrm{n}=37$; either at the subtelomere-telomere junction, $\mathrm{n}=$ 24; or elsewhere, $n=13)$ or TTTTTAGGG $(n=13)$ and TTTTGGG $(n=8)$ (Table 1 and Fig S1B). These three variants were found in at least two independent clones at the same position in the telomere sequence, thus likely representing true low-frequency variants and not sequencing errors. We also detected sequence variants that occurred

Table 1. Frequency of telomeric repeat motifs determined by telomere PCR and sequencing of 32 independent clones.

\begin{tabular}{l|cc} 
Sequence & $\mathbf{n}$ & Frequency \\
\hline TTTTAGGG & 636 & $89.7 \%$ \\
\hline TTTAGGG & 37 & $5.2 \%$ \\
\hline TTTTTAGGG & 13 & $1.8 \%$ \\
\hline TTTTGG & 8 & $1.1 \%$ \\
\hline Others & 15 & $2.1 \%$ \\
\hline
\end{tabular}

only in single clones $(n=15)$ and for which PCR and/or sequencing errors can, therefore, not be ruled out. We conclude that $C$. reinhardtii telomeric repeats are mostly nondegenerate with few lowfrequency variants.

\section{C. reinhardtii telomeres form a protective structure and a subset ends with a $3^{\prime}$ overhang, whereas another subset bears blunt ends}

The protective structure formed by telomeric DNA bound by specific proteins is critical for telomere functions (Palm \& de Lange, 2008). To test the presence of such a structure at $C$. reinhardtii telomeres, we performed a micrococcal nuclease (MNase) digestion of nuclei and asked whether telomere DNA would be protected from its activity. When nuclei were subjected to increasing amounts of MNase, nucleosomal DNA was protected from digestion and migrated at 150 bp based on ethidium bromide staining (Fig 1A, left), as expected (Clark, 2010). Intermediate digestion products migrated in a typical ladder pattern corresponding to di-nucleosomes, trinucleosomes, and higher order structures (Fig 1A, left and middle, asterisks in the lane with one unit of MNase). Strikingly, Southern blot hybridization with a radioactive telomeric probe revealed a diffuse pattern, suggesting that telomeric DNA was protected from MNase digestion in a noncanonical manner (Fig $1 \mathrm{~A}$, right). As a control, the same membrane was stripped and probed for $18 \mathrm{~S}$ rDNA, which showed the canonical nucleosome structure (Fig 1A, middle, asterisks). The size of the protected telomeric DNA was in the range of 200-700 bp, which could correspond to the full telomere length. This result suggests that telomeric DNA might be fully associated with and protected by a noncanonical nucleosomal structure or by other protein complexes, similar to telosomes as observed in yeasts, for example (Wright et al, 1992; Greenwood et al, 2018).

The chromosome end structure determines the protection strategies used to cap the telomere. In many species, telomeres end with a $5^{\prime}$ to $3^{\prime}$ single-stranded overhang, important for the t-loop structure in human telomeres, telomerase recruitment, and binding of specific capping proteins, such as the CST and Ku complexes and POT1 (Palm \& de Lange, 2008; Giraud-Panis et al, 2010; Wellinger \& Zakian, 2012). As it was reported that the Gbp1 protein preferentially binds single-stranded C. reinhardtii telomeric DNA (Johnston et al, 1999), the presence of a 3' overhang would be consistent with a role of Gbp1 at telomeres, possibly protecting them from degradation and fusions similarly to telomere capping proteins in other species. To experimentally test the presence of a $3^{\prime}$ overhang at C. reinhardtii telomeres, we performed primer extension telomere repeat amplification (PETRA) (Heacock et al, 2004). PETRA requires the annealing of an adaptor primer (PETRA-T) to the overhang. After primer extension, the telomere was PCR-amplified using a unique subtelomeric forward primer and a reverse primer (PETRA-A) complementary to a tag sequence present in PETRA-T (Figs 1B and $\mathrm{S1C}$ ). Successful amplification by PETRA is indicative of the presence of a $3^{\prime}$ overhang. Using primers specific for three different telomeres (1R, 9R, and 10R), we found robust amplification of PETRA products in three $C$. reinhardtii strains (CC4350+, T222+, and CC125+), strongly suggesting that these telomeres have a $3^{\prime}$ overhang of at least 12 nucleotides, corresponding to the size of the annealed part of PETRA-T to the overhang (Figs 1B and S1C). Control with prior 
A

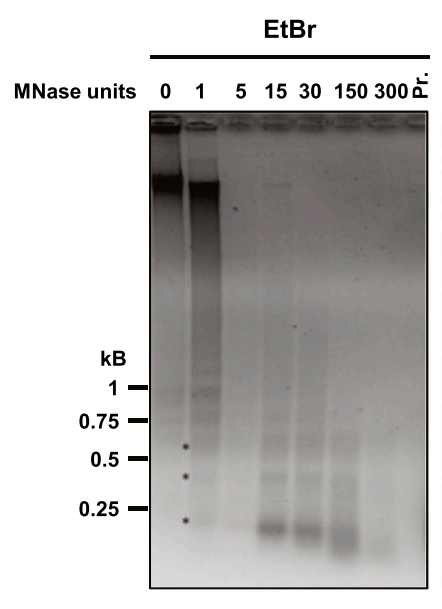

B
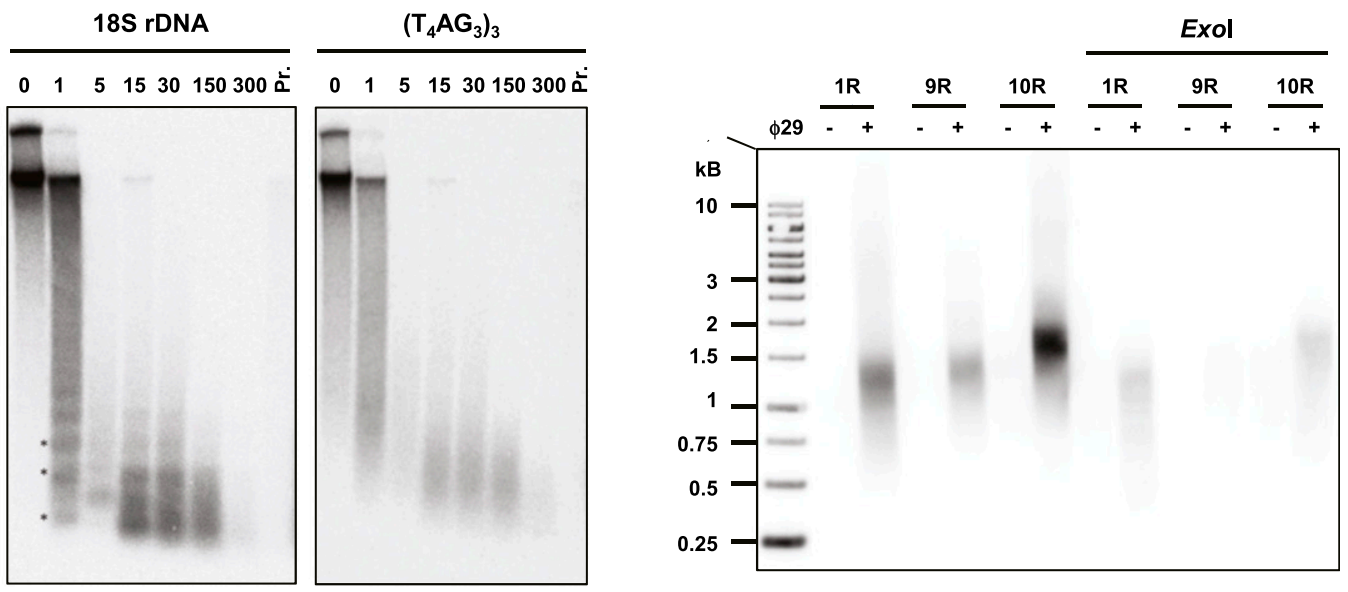

C
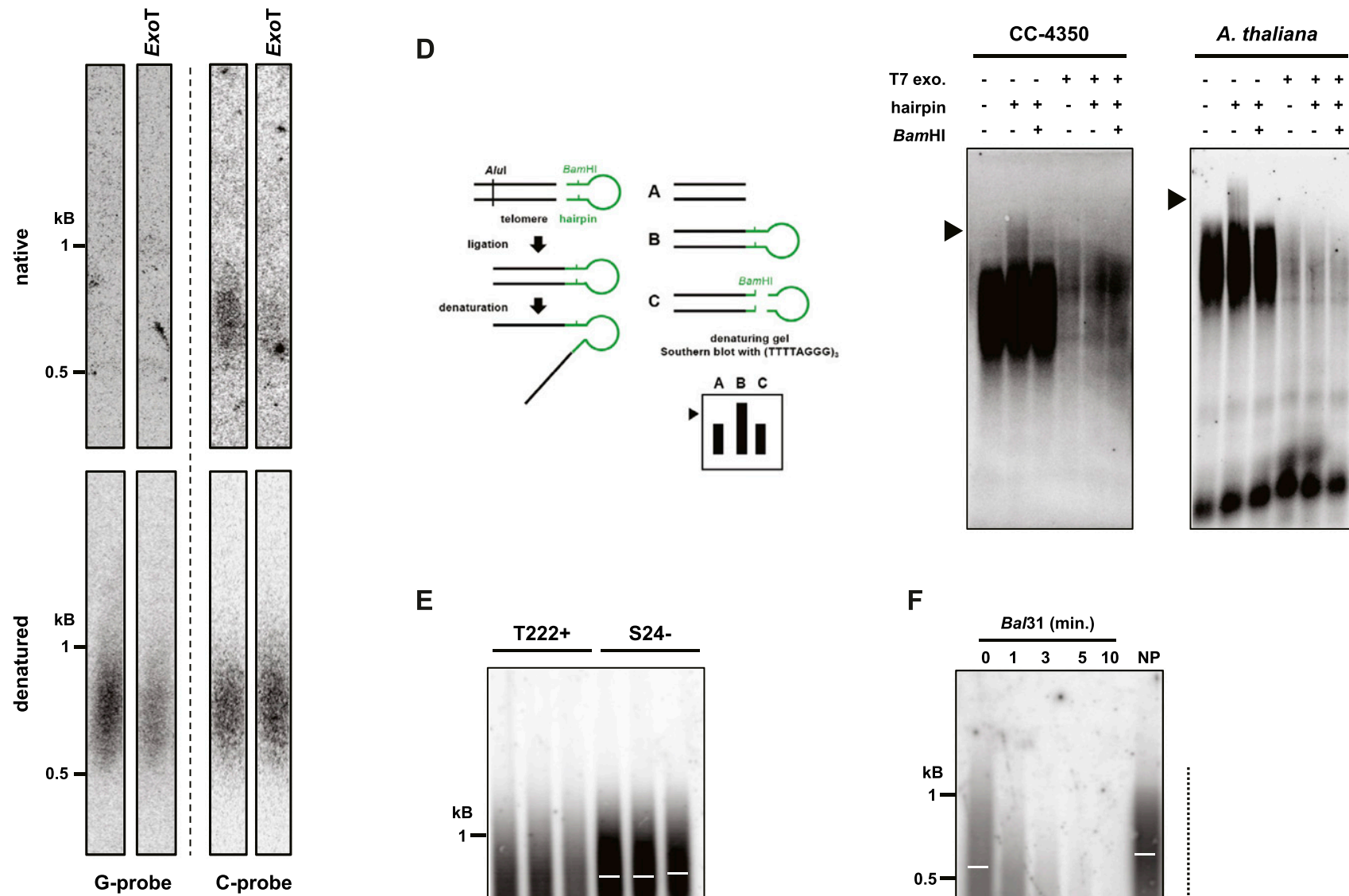

E

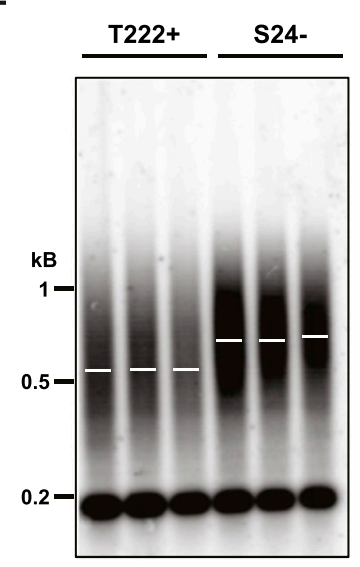

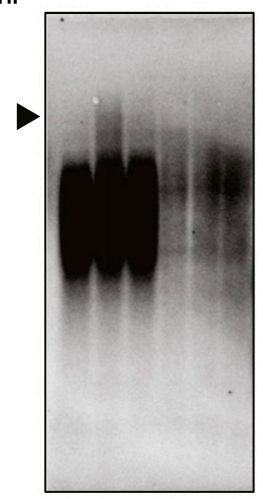

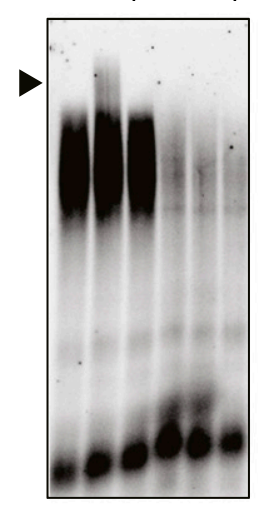

$\mathbf{F}$

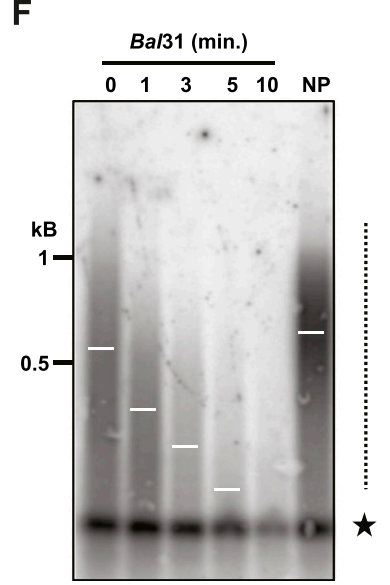

Figure 1. Structural characterization of $C$. reinhardtii telomeres.

(A) Characterization of C. reinhardtii telosomes by MNase digestion of chromatin (left panel; "EtBr": ethidium bromide staining of the migration gel) and Southern analysis with a telomeric specific probe (right panel; (TTTTAGGG) panel). Lanes correspond to increasing amounts of MNase units; "Pr": control where nuclei were digested with 60 MNase units after protein removal. (B) PETRA was used to amplify three different telomeres (1R, 9R, and 10R) from strain CC4350+ and analyzed by Southern blot hybridization using the telomere-specific probe (TTTTAGGG) (see also Fig S1C). Negative controls include omission of the primer extension step by $\Phi 29$ polymerase and pretreatment of the samples with Exol. (C) Upper panel: Native 
treatment of the samples with exonuclease I (Exol) showed a decreased PETRA efficiency, indicating that the PETRA-T primer indeed requires $3^{\prime}$ overhang for annealing. The presence of a 3' overhang at telomeres was further confirmed by native in-gel hybridization assay, which detected exonuclease T (ExOT)-dependent singlestranded DNA on the G-rich strand of the telomeres of strain T222+, but not on the C-rich strand (Figs $1 \mathrm{C}$ and S1D).

As it was shown that a subset of $A$. thaliana telomeres displays blunt ends instead of 3' overhangs (Kazda et al, 2012), we asked whether blunt-ended telomeres also exist in C. reinhardtii because the PETRA and in-gel experiments do not exclude this possibility. To test this, we applied the hairpin assay, which was successfully used in A. thaliana to detect blunt-ended telomeres (Kazda et al, 2012). Briefly, a synthetic hairpin DNA can be ligated to both strands of the telomeres, only if they are blunt ended. After digestion with Alul at a site in the subtelomeres, the ligated products migrate as a doublesized fragment compared with the unligated control in denaturing conditions. Cleavage of the ligated product by BamHI, using a restriction site designed in the hairpin, can then show that the slow migrating product was indeed generated by ligation to the hairpin (Fig 1D, left). Ligation products of a higher molecular weight were clearly detected using this hairpin assay in C. reinhardtii strain CC4350+ as in A. thaliana (Fig 1D, right, arrows) and they were abolished by cleaving the hairpin by BamHI or pretreatment with $\mathrm{T} 7$ exonuclease that generates $3^{\prime}$ single-stranded DNA protrusions at DNA ends. This demonstrates that a fraction of telomeres in $C$. reinhardtii is blunt ended.

Taken together, our structural analysis of telomeres indicates that as in A. thaliana, chromosome ends in C. reinhardtii are composed of two subsets, one ending with a $3^{\prime}$ overhang and the other with a blunt end.

\section{Terminal restriction fragment (TRF) analysis of $C$. reinhardtii telomeres}

To study telomere length distributions and their possible regulations, we optimized a TRF analysis for $C$. reinhardtii to accurately measure telomere length from populations of cells.

We first measured telomere length in three independent biological replicates of strains T222+ and S24-, two isogenic reference strains differing only in their mating type (Gallaher et al, 2015). We found that telomere fragments spread as a smear over a large range of lengths, from $\sim 200$ to $\sim 1,200 \mathrm{bp}$ (Fig 1E). The two strains displayed a significant difference in their average telomere length ( mean \pm SD: T222+ = $539 \pm 54$ bp; $N=18$ and S24- = $710 \pm 12$ bp; $N=5$ ). To demonstrate that the detected smeary signal corresponded to terminal fragments, we digested the genomic DNA (gDNA) with exonuclease Bal31 before restriction digestion (Fajkus et al, 2005) and indeed observed that with increasing incubation times with Bal31, the signal progressively decreased in size until it nearly disappeared after 10 min (Figs $1 F$ and S1E). The migration of a band at 200 bp was not altered even with the longest Bal31 treatment, indicating that it stemmed from interstitial telomere repeats located within the genome. Because this sharp band did not cross-react with a probe targeting TG microsatellite sequences (Fig S1F), it most probably corresponded to bona fide telomere sequence-containing region(s) of the genome and not to nonspecific cross hybridizations.

\section{Telomere length distribution is stable in different standard growth conditions}

C. reinhardtii has been widely used as a model organism to study photosynthetic processes because of its ability to grow in different metabolic regimes (Harris, 2009). Under strictly phototrophic conditions (minimum medium in the light), photosynthesis is the only metabolic process providing ATP and reducing power to growing cells. In strictly heterotrophic conditions in the dark, $C$. reinhardtii can survive by respiring the acetate contained in Trisacetate-phosphate (TAP) medium. In mixotrophic conditions, that is, TAP medium in the light, cells use a combination of photosynthesis and respiration to grow. Because in other organisms, environmental conditions can regulate telomere length (Walmsley \& Petes, 1985; von Zglinicki, 2000; Epel et al, 2004; Romano et al, 2013), we asked whether telomeres vary in length and/or size distributions in response to different standard growth conditions.

We first tested whether cells displayed different telomere lengths during a standard growth kinetic in TAP medium, from inoculation to exponential and then stationary phase, sampled at different time points over a period of $8 \mathrm{~d}$. We observed no significant difference in telomere length between the samples (Fig 2A). Prolonged incubation in stationary phase for up to $15 \mathrm{~d}$ also did not affect telomere length (Fig 2B). Thus, telomere length was not altered either in exponential growth in replete medium or in the absence of growth, during nutrient depletion, and with any other property of saturated cultures, even over a prolonged period.

We also asked whether stimulating cell growth could affect telomere length. Because of the multiple fission mode of cell division of C. reinhardtii (Cross \& Umen, 2015), actively growing cells might spend less time in each phase of the cell cycle, and we reasoned that on average, telomerase might thus be less active. To test this hypothesis, a TAP culture was constantly maintained in exponential growth phase by serial dilutions over a period of $10 \mathrm{~d}$. Telomere length did not significantly change (Fig 2C), and therefore, high division rate did not affect telomere length or distribution.

Finally, we checked telomere length distributions in cultures grown in either strictly phototrophic, strictly heterotrophic, or

in-gel hybridization assay of telomeres from strain T222+ using a G-probe (oT0958, left) or a C-probe (oT0959, right). Most of the native signal, when hybridized with the C-probe, was absent when the gDNA was pretreated with ExoT. Lower panel: The native gel was then denatured and transferred to a membrane, which was then hybridized with the same probes. The uncropped gel and membrane are shown in Fig S1D. (D) Hairpin ligation assay on C. reinhardtii CC4350+ strain and A. thaliana. A scheme of the assay is shown (left). Digestion with BamHI, which removes the ligated hairpin and pretreatment with T7 exonuclease ("T7 exo."), which resects the $5^{\prime}$ end of a duplex DNA, are used as controls. (E) T222+ and S24- strains were subcloned and three subclones were independently grown in liquid culture until stationary phase and subsequently analyzed by TRF Southern blot hybridization. (F) gDNA was subjected to Bal31 digestion for 0 to 10 min. Digested products were column-purified and then processed for TRF analysis. 0: no Bal31 digestion, but gDNA was column-purified before digestion by the restriction enzymes. NP: gDNA was directly analyzed by TRF, with No column Purification. Dashed line: smear corresponding to telomeres. Star: Bal31-insensitive band, corresponding to interstitial telomeric repeat (see also Fig S1E). 
A
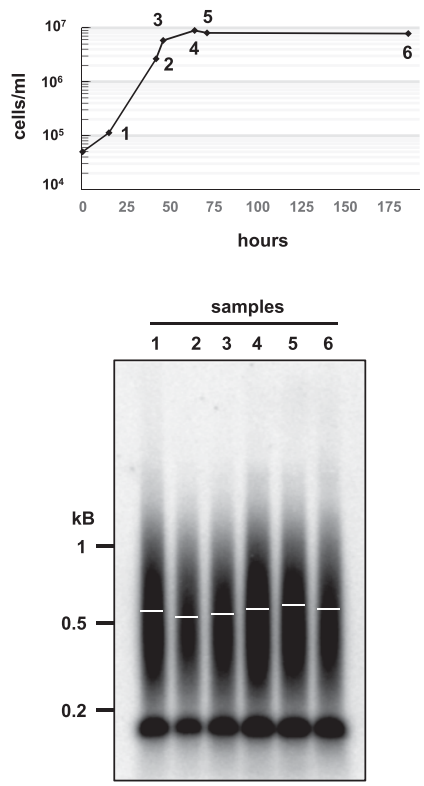

B
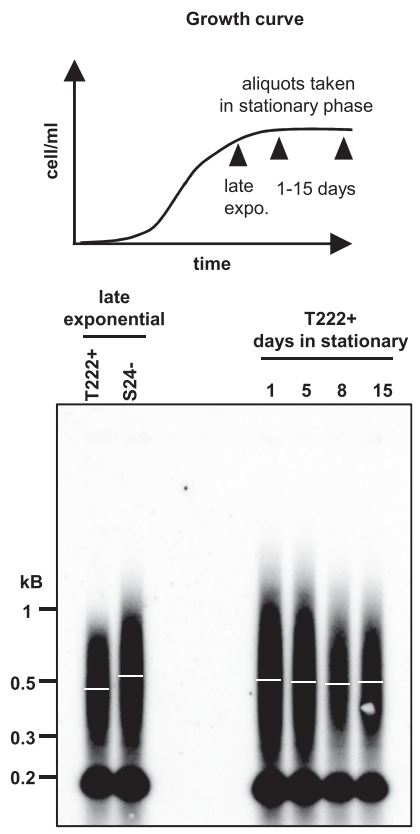

C
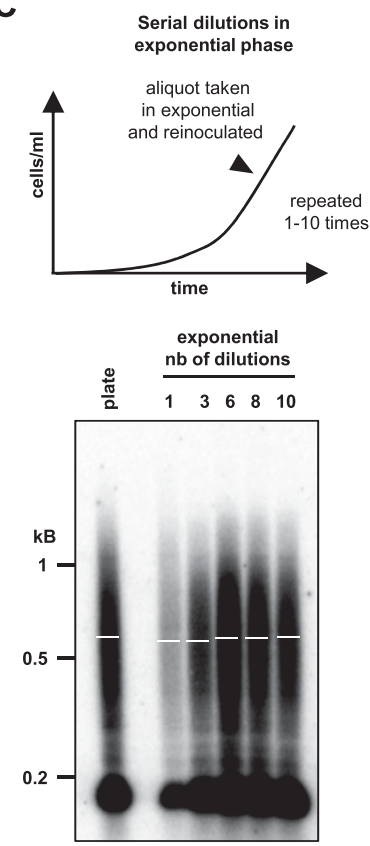

D

TAP: Tris-Acetate Phosphate medium MIN: Minimum medium LL: $10 \mu \mathrm{E} \cdot \mathrm{m}^{-2} \cdot \mathrm{s}^{-1}$

Dark: heterotrophy TAP, LL/HL: mixotrophy MIN, HL: phototrophy

Figure 2. Telomere length distribution is stable under various growth conditions.

(A) Telomere length distributions of T222+ strain at different growth stages of liquid cultures. T222+ cells were harvested at early exponential (1), mid-exponential (2), late exponential (3), and early $(4,5)$ and late (6) stationary phases and analyzed by TRF Southern blot hybridization. (B) Telomere length distributions of prolonged cultures in stationary phase. The cells were harvested after 1, 5, 8, and $15 \mathrm{~d}$ after reaching stationary phase and compared with late exponential cultures. (C) Telomere length distributions of serial dilutions of rapidly growing cells. A liquid culture of T222+ cells was grown to exponential phase ( $2 \times 10^{6}$ cells/ml), a sample of cells was harvested and the remaining cells diluted with fresh media to $5 \times 10^{4}$ cells $/ \mathrm{ml}$. This serial dilution was repeated 10 times. Samples corresponding to dilutions 1,3 , 6,8 , and 10 were then analyzed by TRF Southern blot hybridization. Plate: Cells were directly scraped from 1-wk-old streaks on TAP Petri dishes, without liquid culture. (D) Telomere length distributions in different metabolic growth conditions. The cells were grown for $6 \mathrm{~d}$ to stationary phase either in heterotrophic conditions in TAP medium in the dark, in mixotrophic conditions in TAP medium in low (LL) or higher light (HL), or in pure photo-autotrophic conditions in minimum (MIN) medium under HL.

mixotrophic conditions for $7 \mathrm{~d}$ in liquid medium ( 20 population doublings) but found no significant difference between the conditions (Fig 2D). As telomeres might reach a new steadystate level with a slower kinetic, we repeated the experiment over a period of $60 \mathrm{~d}(200$ population doublings) but again did not detect changes in telomere length regardless of the growth conditions (Fig S2).

These experiments demonstrated that $C$. reinhardtii has an active telomere maintenance mechanism and that telomere length distribution is robust with regards to perturbation in metabolic regimes under a variety of standard laboratory growth conditions.

\section{C. reinhardtii reference strains show important differences in telomere length and size distributions}

Even though telomere length distribution was very stable under different growth conditions for a given strain (Fig 2), we did observe a reproducible and significant difference in mean telomere length between the three laboratory reference strains CC4350+, T222+, and CC125+ by PETRA (Figs 1B and S1C) and between T222+ and S24- by TRF Southern blot (Fig 1E). We thus wondered if related $C$. reinhardtii strains displayed inter-strain differences in telomere length distribution. To test this, we took advantage of the recent sequencing of many closely related reference strains widely used in different laboratories across the world and which display up to 2\% genetic divergence (Gallaher et al, 2015). We performed TRF analysis on 12 related $C$. reinhardtii strains to characterize their telomeres (Figs 3A and S3A). Strikingly, steadystate telomere lengths were highly variable from strain to strain, ranging from $378 \pm 24 \mathrm{bp}($ mean $\pm \mathrm{SD}, \mathrm{N}=4)$ in $\mathrm{CC} 125+$ to $3.2 \pm 1.1 \mathrm{~kb}$ $(\mathrm{N}=3)$ in $\mathrm{cw} 15 . J 14+$, encompassing nearly one order of magnitude (Fig 3B). Telomere length did not correlate with genome divergence (genetically close strains are depicted with the same color), and we did not find any obvious genomic region, as described by Gallaher et al (2015), that would cosegregate with longer or shorter telomeres. In particular, neither the mating type nor the presence or absence of a cell wall correlated with telomere length variations. The average telomere length in strain cw15.J14+ was particularly striking, and we asked whether the signal could stem from internal telomere repeats. A Bal31 exonuclease treatment time course showed the signal decreasing in size demonstrating that this signal indeed corresponded to terminal repeats (Fig S3B, right). In addition to length variations, some strains, such as CC503+ and CC1010+, displayed multimodal telomere length distributions (Figs $3 \mathrm{~A}$ and $\mathrm{B}$, and $\mathrm{S} 3 \mathrm{~A}$ ), and the multiple peaks corresponded to terminal fragments and not internal ones (Fig S3B, left).

Interestingly, the interstitial band at $\sim 200 \mathrm{bp}$, which was present in 11 tested $C$. reinhardtii reference strains was absent 
A

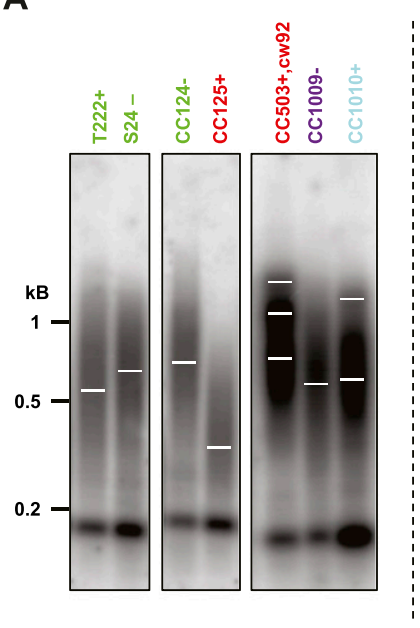

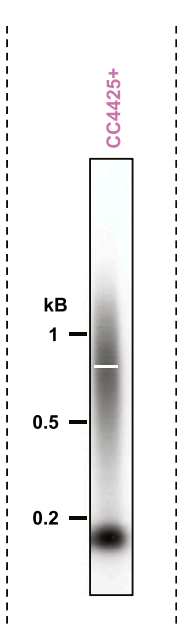

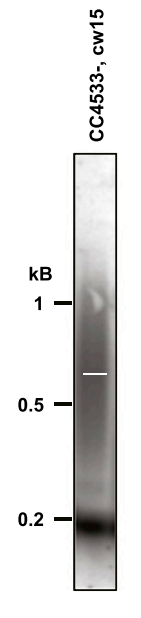

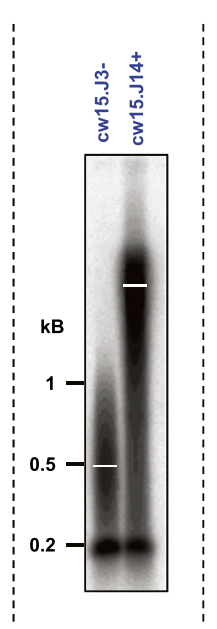

B

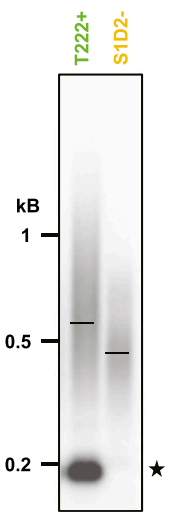

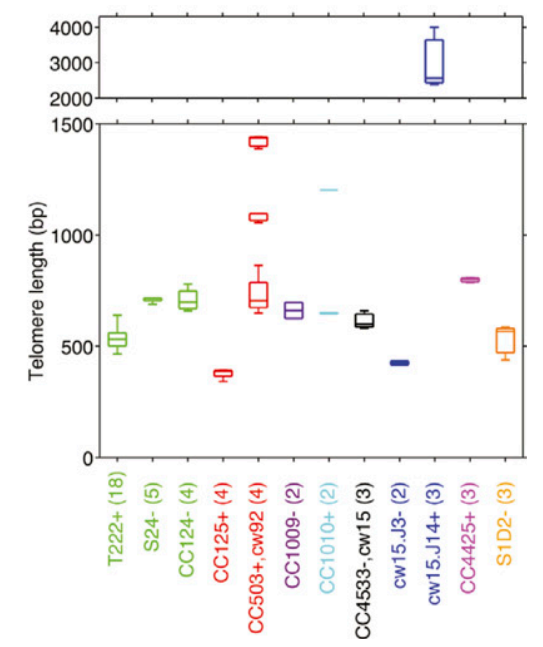

Figure 3. Vast differences in telomere length distributions in C. reinhardtii reference strains.

(A) Telomeres of recently sequenced C. reinhardtii reference strains (Gallaher et al, 2015) were analyzed by TRF analysis. Strains sharing the same name color are closely related genetically, whereas strains with different colors are more divergent. Dashed vertical lines indicate independent gels. Star: S1D2- strain does not display the band at 200 bp. cw15 and cw92 indicate mutations that led to cell wall-less strains. (B) Mean and SD of telomere length for each strain as calculated by analysis of Southern blots from the indicated number of independent biological replicates $(\mathrm{N})$.

from the S1D2- (CC2290-) strain (Fig 3A, star). S1D2- is an interfertile but divergent $C$. reinhardtii strain, often used for genetic mapping purposes (Gross et al, 1988; Vysotskaia et al, 2001). Thus, the interstitial telomere sequence might have emerged in a subset of $C$. reinhardtii species or conversely might have been lost in S1D2-.

\section{Identification of the gene encoding the catalytic subunit of telomerase}

Telomerase is a holoenzyme comprising at least a reversetranscriptase catalytic subunit and a template RNA, which are sufficient for in vitro telomerase activity (Lingner et al, 1997a). These core actors are associated with multiple other proteins, required for its recruitment, processivity, and regulation (Lewis \& Wuttke, 2012). As the catalytic subunit of telomerase (e.g., hTERT in human, AtTERT in A. thaliana, and Est2 in Saccharomyces cerevisiae) is conserved, we sought to identify the gene encoding this subunit in C. reinhardtii and to characterize the contribution of telomerase to telomere length maintenance. Nucleotide BLAST searches in C. reinhardtii genome failed to find similarity to most of the shelterin or shelterin-like genes and telomerase-associated genes from human, A. thaliana, and S. cerevisiae, except for CBF5 from A. thaliana (also CBF5 in C. reinhardtii), corresponding to the dyskerin gene.

Gene model Cre04.g213652 of the C. reinhardtii nuclear genome (Phytozome v5.5; https://phytozome.jgi.doe.gov/pz/\#) has a predicted $\mathrm{N}$-terminal part of the corresponding protein showing partial sequence similarity with the RNA-binding domain of telomerase from a number of organisms (Fig 4A). However, the available gene model extends over $25 \mathrm{~kb}$, contains 28 introns and is predicted to encode a 5,019-aa protein, much larger than telomerase from $A$. thaliana (1,123 aa), maize (1,188 aa), iris (1,295 aa), and rice (1,261 aa), overall suggesting that the current gene model is probably incorrect. In addition, two sequencing gaps and the presence of TG and CCAC satellites both in introns and exons cloud the structure of the putative gene. Although expressed sequence tags from cDNA libraries supported the validity of some parts of the conserved $5^{\prime}$ and $3^{\prime}$ regions, no expressed sequence tag was found for the central part of the gene model in the available $C$. reinhardtii expression libraries. As stated above, nucleotide sequence alignments failed to detect similarity with telomerase catalytic subunit genes of other organisms. We thus performed PSI-Blast alignments of the C-terminal protein domain of the putative $C$. reinhardtii telomerase with telomerase from plants using PRALINE (http:// www.ibi.vu.nl/programs/pralinewww). The alignments showed strong similarity to the $\mathrm{C}$-terminal catalytic reverse-transcriptase domain of $A$. thaliana $\left(e-v a l u e=3 \times 10^{-36}\right)$, maize $\left(e-v a l u e=4 \times 10^{-35}\right)$, iris $\left(e-v a l u e=1 \times 10^{-36}\right)$, and rice $\left(e-v a l u e=3 \times 10^{-24}\right)($ Fig 4B). The conserved $\mathrm{C}$ motif $(\mathrm{mC}$ ) in organisms ranging from $\mathrm{S}$. cerevisiae to $A$. thaliana and humans, including two critical aspartates for telomerase catalytic activity (Lingner et al, 1997b; Nakamura et al, 1997; Oguchi et al, 1999) showed strong sequence conservation with a corresponding motif in the putative $C$. reinhardtii protein (Fig 4B and $C$ ). Motif $E$ ( $m E$ ) was conserved to a lesser degree, whereas no clear conservation of motifs mA, mD, and motif 1 and 2 (Lingner et al, 1997b; Oguchi et al, 1999) was found in the predicted C. reinhardtii protein. Other well-conserved regions in the C-terminal part with no assigned motif are also depicted in Fig 4B.

To demonstrate that the genomic region Cre04.g213652 indeed contains the gene encoding the catalytic subunit of telomerase of $C$. reinhardtii, we selected three strains harboring insertions of the paromomycin resistance cassette within the putative gene from the recently created CliP library of mapped insertional mutants (Li et al, 2016) (https://www.chlamylibrary.org) (Fig 4A). LMJ.RY0402.077111 has an insertion in a putative intron near the region encoding the 
A

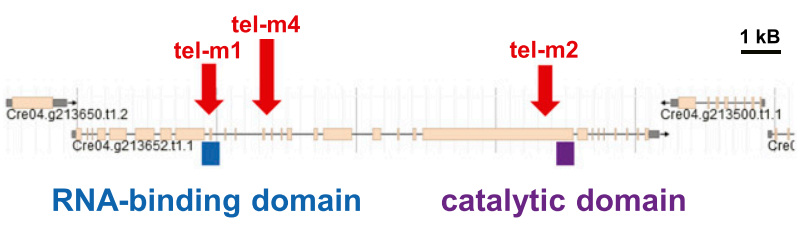

B
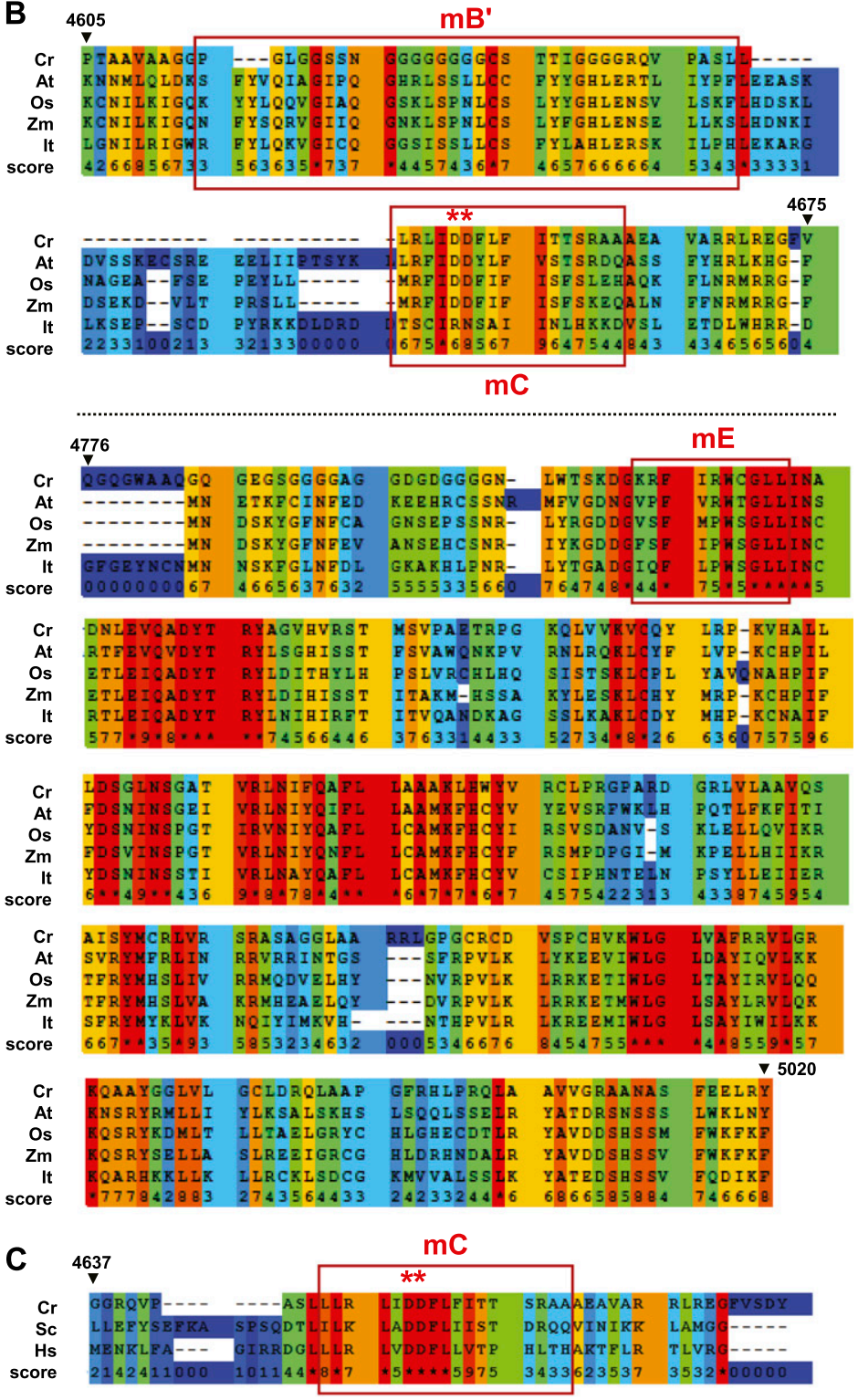

$\mathrm{mE}$
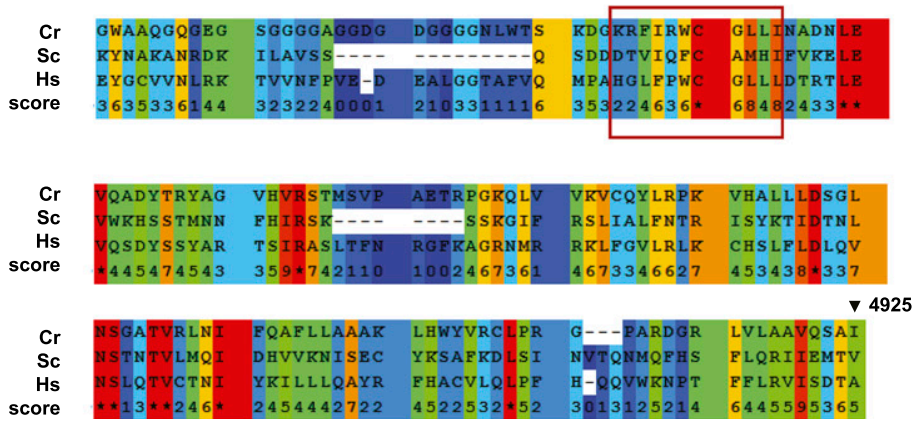

Figure 4. Identification of the CrTERT gene encoding the catalytic subunit of telomerase in $C$. reinhardtii.

(A) The protein corresponding to the predicted gene model Cre04.g213652.t1.1 of the available C. reinhardtii nuclear genome harbors an annotated $\mathrm{N}$ terminal domain with significant similarities to the RNA template-binding domain of telomerases from other organisms. The C-terminal domain shows strong similarities with the catalytic domain of this enzyme in other organisms. Mutants tel-m1 (LMJ.RY0402.077111) and tel-m2

(LMJ.RY0402.209904) from the CliP library have reported insertions in either the RNA-binding or the catalytic domain, respectively. Mutant tel-m4 (LMJ.RY0402.105594) has an insertion in between these two domains. (B) PSIblast alignments show strong amino-acid sequence similarity of the catalytic domain of telomerases from many organisms with the putative $C$. reinhardtii protein. Similarity score ranges from 0 (light blue) to 9 and * (red) indicates identity. The motifs $\mathrm{B}^{\prime}, \mathrm{C}$, and $\mathrm{E}\left(\mathrm{mB}^{\prime}, \mathrm{mC}\right.$, and $\left.\mathrm{mE}\right)$ described by Lingner et al (1997b), Ogushi et al (1999) show strong conservation in C. reinhardtii, including two catalytic aspartates, essential for telomerase function in other organisms (red asterisks). Conservation can also be observed downstream of mE between CrTERT and the other telomerases. (C) The $\mathrm{mC}$ motif of $C$. reinhardtii shows strong sequence similarity with the $\mathrm{mC}$ motif containing two catalytically essential aspartates in yeast and human telomerases (Lingner et al, 1997b; Ogushi et al, 1999). Cr, C. reinhardtii; At, A. thaliana; Os, O. sativa; Zm, Z. mays; It, I. tectorum; Sc, S. cerevisiae; Hs, H. sapiens. 
putative RNA-binding domain of the gene and was named tel-m1. LMJ.RY0402.209904 has an insertion in the putative CDS of the putative catalytic C-terminal domain and was named tel-m2. LMJ.RY0402.105594 has an insertion in an intron in a nonconserved region between these two domains and was named telm4. Although the insertions in these three mutants were already mapped by the work of Li et al (2016) with a confidence of 95\% for tel-m1 and tel-m4 and 73\% for tel-m2, we verified that all three mutants indeed had the insertion at the predicted loci, using PCR with primers targeting the gene and/or the inserted paromomycin resistance marker (Fig S4A and B). For all three mutants, the obtained PCR products were gel-excised, sequenced, and shown to correspond to the expected genomic region. We also backcrossed mutants tel-m1 and tel-m2 with the paromomycin-sensitive T222+ strain and analyzed the segregation of the paromomycin resistance phenotype in tetrads after germination of the diploids (Fig S4C). Paromomycin resistance systematically segregated with a 2:2 ratio in the haploid offspring, suggesting that the functional marker was not inserted at multiple loci in the genome. Correct 2:2 segregation of the mating locus in the offspring of the tetrads was checked by PCR (Fig S4D).

We then analyzed the telomere length of the three mutant strains. All three mutants showed significantly shorter telomeres when compared with the parental CC4533- strain used by Li et al (2016) to construct the CliP library (Figs 5A and S5A; mean \pm SD, tel$\mathrm{m} 1: 373 \pm 25 \mathrm{bp}, \mathrm{N}=4$, tel $-\mathrm{m} 2: 383 \pm 30 \mathrm{bp}, \mathrm{N}=4$, and tel $-\mathrm{m} 4: 387 \pm 12 \mathrm{bp}$, $\mathrm{N}=2$, compared with CC4533-: $614 \pm 41 \mathrm{bp}, \mathrm{N}=3$ ). We verified that the shorter telomere length in mutants tel-m1, tel-m2, and tel-m4 was not simply due to the transformation protocol used to generate

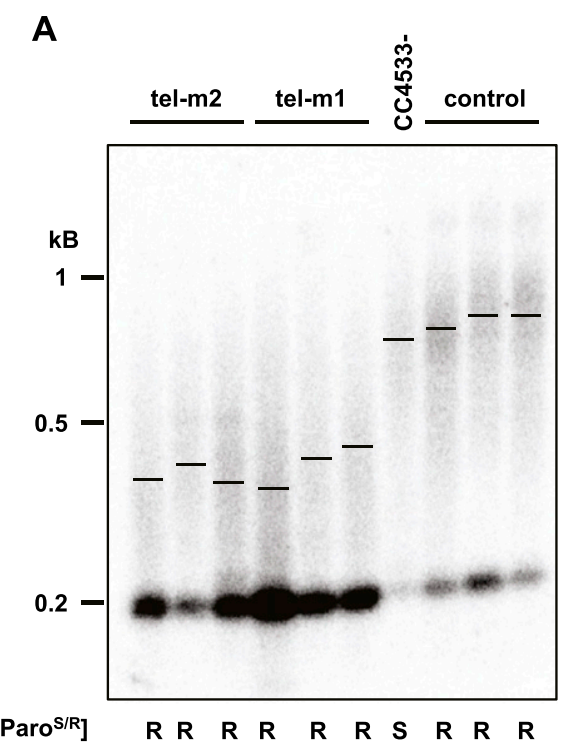

C

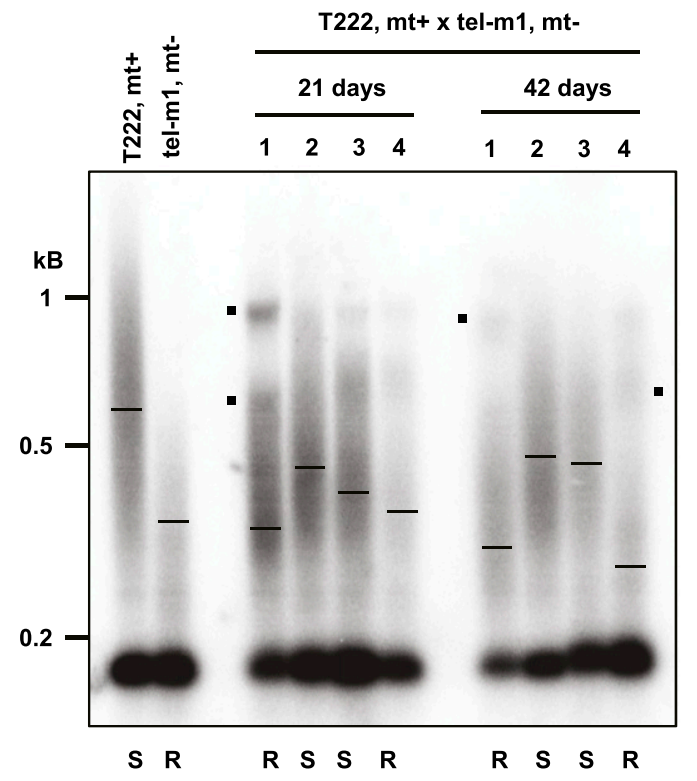

B

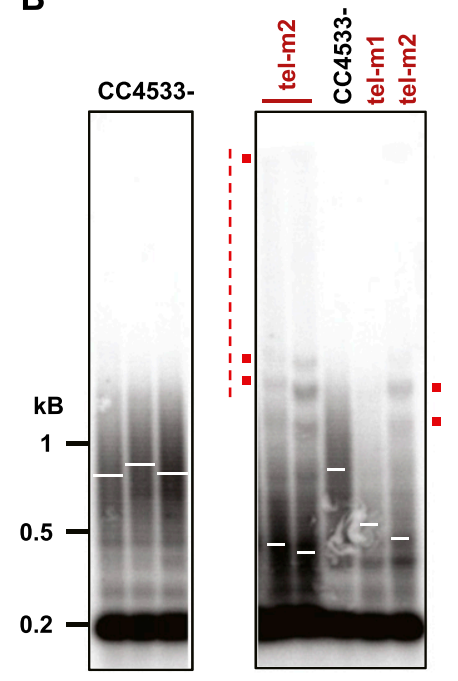

Figure 5. Insertional mutants of the CrTERT gene have shorter telomeres.

(A) Mutants tel-m1 and tel-m2 have shorter telomeres in TRF analyses (three independent subclones are shown). Control: mutant LMJ.RY0402.239308 from the CliP library, which has an insertion in a gene unrelated to CrTERT. Paromomycin resistance phenotype is indicated

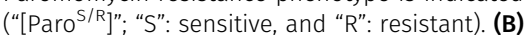
Prolonged liquid cultures of telomerase mutants lead to rearranged TRF patterns. Cells were cultured in liquid medium for 2 mo before TRF analysis. Additional bands and slow-migrating DNA molecules (red dots and dotted vertical line, respectively) are indicated for tel$\mathrm{m} 1$ and tel-m2 and are not present in the CC4533reference strain TRF pattern. (C) Tetrad analysis of the cross between tel-m1 and T222+ shows a 2:2 cosegregation of paromomycin resistance and shortened telomeres after 21 and $42 \mathrm{~d}$ after the cross (see also Fig S5B). Mating types "mt+" and "mt-" are indicated. (D) Tetrad analysis of the cross between tel$\mathrm{m} 2$ and $\mathrm{T} 222+$ shows a 2:2 cosegregation of paromomycin resistance and shortened telomeres after $\sim 80 \mathrm{~d}$ after the cross (see also Fig S5C). Mating types " $\mathrm{mt}+$ " and "mt-" are indicated.
D

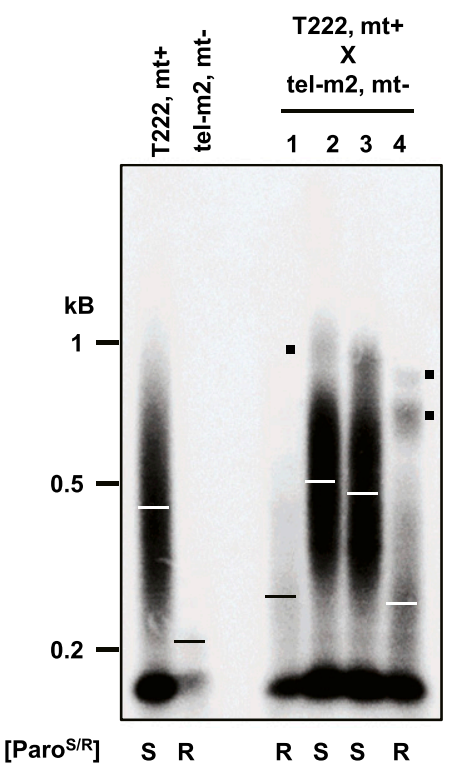


the CliP library or to the insertion of the paromomycin marker itself. Telomere length was measured in another mutant from the CliP library, harboring an insertion elsewhere in the genome (on chromosome 1), and was comparable with the parental CC4533strain (Fig 5A, "control" and Fig S5D).

We conclude that although gene model Cre04.9213652 might be wrong in its predicted structure and will require further study to be corrected, this genomic region indeed harbors the gene encoding for the catalytic subunit of telomerase in C. reinhardtii, and we propose to rename it CrTERT.

\section{Telomere rearrangement and maintenance in long-term cultures of telomerase mutants}

Because telomeres shortened in telomerase-negative cells, we wondered whether the cells would experience replicative senescence after an extended period of growth, when telomeres reach a critically short length. We thus grew the telomerase mutants tel-m1 and tel-m2 as well as the reference strain for 2 mo ( 200 population doublings), with dilutions into fresh TAP medium every $5 \mathrm{~d}$. We did not observe CrTERT mutant cultures dying out or any obvious growth defect at any time point. However, TRF analysis of these long-term cultures of tel-m1 and tel-m2 showed alterations of their telomere length distribution (Fig 5B, compare with Fig 5A): additional discrete bands appeared at sizes above $1 \mathrm{~kb}$ (red dots), and a signal that extended up to the wells was detected (vertical red line). Interestingly, the three independent cultures of the tel-m2 mutant gave similar but distinct patterns with respect to the discrete bands and the high molecular weight signal. The tel-m1 mutant also displayed some additional bands, albeit not to the extent of tel-m2. Overall, these altered TRF patterns observed in prolonged cultures of telomerase mutants are reminiscent of TRF patterns observed for cells with telomerase-independent maintenance pathways (e.g., type II survivors of telomerase-negative yeast cells or alternative lengthening of telomeres (ALT)-like telomerase-negative cancer cells. See the Discussion section.)

\section{Telomeres shorten progressively in telomerase mutants}

The initial Clip telomerase mutants might have accumulated additional, potentially suppressor, mutations, which could interfere with the proper assessment of the mutant phenotype. Importantly, the presence of suppressor mutations could explain why these mutants did not show any discernible growth defects in standard growth conditions or any sign of senescence after prolonged culture.

To outcross potential suppressor mutations and gain a kinetic perspective on telomere shortening in the telomerase mutants, we backcrossed mutants tel-m1 and tel-m2 with a wild-type strain of opposite mating type (T222+) and, after germination of the diploids, studied the telomere length distribution of the obtained tetrads. Backcrossing a mutant cell with a telomerase-positive strain should allow telomerase to elongate the shortest telomeres brought in by the mutant strain. The subsequent meiosis would then shuffle the chromosomes and the telomeres in the four haploid cells, independently of the mutant or wild-type status of the telomerase gene. We thus expect that immediately after meiosis in the diploid, the four haploid cells would have similar and nearly wild-type average telomere length. After culture, the telomere length in the four progenies should vary according to the status of the CrTERT gene.

Strikingly, measurement of telomere length in the four haploid progenies of the tel-m1- $x$ T222+ cross after $21 \mathrm{~d}$, the earliest time point we could obtain, showed that two of them displayed on average longer telomeres and the other two shorter telomeres, which corresponded to the telomerase mutants as assessed by paromomycin resistance (Fig 5C, "21 d"). After 21 more days, the telomeres of the telomerase-positive cultures maintained or increased their average length, whereas the telomerase-negative cultures displayed further shortening of their telomeres (Fig 5C, " $42 \mathrm{~d}$," and Fig S5B). A similar result was observed for the progenies of the cross tel-m2- x T222+ (Figs 5D and S5C). Telomere length could not be assessed in the dormant diploid state, as diploid cells could not be grown. These results strongly argued against the possibility that the shorter telomeres observed in tel-m1 and tel-m2 were due to additional mutations in the genome because they would not necessarily have co-segregated with the paromomycin marker. We also noted the presence of other bands and peaks in the smear, which were likely the result of segregating parental telomeres of very different lengths during meiosis (black dots in Figs 5 C and D, and S5B).

Although no growth defect was observed for the initial tel-m1 and tel-m2 mutants, analysis of the haploid progeny from backcrosses between tel-m1 and tel-m2 with the wild-type T222+ strain ( $n=4$ independent tetrads, with eight telomerase-negative haploids) showed that four of the eight telomerase-negative haploid progenies experienced growth defects and then massive cell death, typical of replicative senescence (highlighted in red in the table of Fig S5E). Strikingly, for each of these four telomerase-negative progenies that experienced massive cell death, some cells managed to form colonies again at very low frequency (Fig S5E, left) and thus corresponded to postsenescence survivors. The four other telomerase-negative haploid progenies did not display any growth defect (highlighted in green in the table of Fig S5E). Individual colonies of postsenescent survivors kept on solid media showed cycles of moderate growth and subsequent cell death. This complex and dynamic survivor phenotype will be investigated in future studies.

\section{Discussion}

In this study, we provide a detailed molecular characterization of $C$. reinhardtii telomeres by investigating their sequence, end structure, and length distribution. We also identify CrTERT, the gene encoding the catalytic subunit of telomerase, and find that mutants of this gene experience telomere shortening and can enter replicative senescence.

\section{Telomere sequence and end structure}

Because telomere-bound proteins specifically interact with telomere sequences (Palm \& de Lange, 2008; Fulcher et al, 2014), the 
variability of telomeric repeat motif can have functional consequences (Arneric \& Lingner, 2007; Marzec et al, 2015). C. reinhardtii telomeric repeats are mostly nondegenerate, with few lowfrequency variants, notably repeats of the canonical $A$. thaliana type (TTTAGGG), possibly as a remnant of the ancestral motif in the green lineage (Fulnečková et al, 2012). The overall low occurrence of variants (Table 1) suggests that $C$. reinhardtii telomerase is a highfidelity reverse transcriptase, in contrast to telomerase from other unicellular eukaryotes such as S. pombe or S. cerevisiae (Zakian, 1995).

Analysis of the available genome sequences of $C$. reinhardtii strains and our TRF analysis suggests the presence of interstitial telomeric repeats, which might be due to chromosome end-to-end fusion over the course of evolution (Meyne et al, 1990; Azzalin et al, 2001; Uchida et al, 2002; Gaspin et al, 2010; Aksenova et al, 2015). But whether they might have any functional role, for example, as binding site for transcription factors (Platt et al, 2013) remains to be investigated.

The protective function of telomeres is not solely provided by their sequence and associated proteins but also by the structure formed at the termini. Similar to A. thaliana, C. reinhardtii telomeres seem to comprise two subsets with different end structures: $3^{\prime}$ overhangs and blunt ends. The latter might correspond to telomeres replicated by the leading strand synthesis, which naturally generates a blunt end. Although we cannot exclude that the structures we detect might represent transient blunt ends that then undergo further processing, the very short relative duration of the $S$ phase in C. reinhardtii cell cycle rather suggests that the blunt ends are stable structures. Although 3' overhangs are common to many eukaryotic species, blunt ends have been observed in plants, mostly in angiosperms but not in the moss Physcomitrella patens (Kazda et al, 2012). Our discovery that the blunt ends are present in green algae suggests that this structure is of much older evolutionary origins than previously thought.

\section{Intra-strain stability and inter-strain variations in telomere length distribution}

Telomere length is regulated by multiple pathways, as shown by exhaustive screens performed in S. cerevisiae (Askree et al, 2004; Gatbonton et al, 2006; Ungar et al, 2009; Chang et al, 2011), including nucleic acid metabolism, DNA replication, chromatin modification, and protein degradation, among others. In addition, telomere length is also sensitive to both internal and environmental cues (Walmsley \& Petes, 1985; von Zglinicki, 2000; Epel et al, 2004; Cetin \& Cleveland, 2010; Romano et al, 2013; Fulcher et al, 2014; Millet et al, 2015; Millet \& Makovets, 2016). We found no change in telomere length distribution when $C$. reinhardtii cells were grown in a wide variety of physiologically relevant laboratory conditions, including growth phases, carbon sources, and light conditions. Although we cannot exclude that other harsher growth conditions or internal signaling (e.g., DNA damage or replication stress) might induce an alteration in telomere length or structure, this result suggests that the mechanisms maintaining telomere length homeostasis are highly robust and efficient.

In stark contrast, closely related strains of C. reinhardtii displayed very different telomere length profiles, similar to variations observed in different strains, isolates, or ecotypes of other species (Walmsley \& Petes, 1985; Burr et al, 1992; Zhu et al, 1998; Shakirov \& Shippen, 2004; Raices et al, 2005; Liti et al, 2009; Fulcher et al, 2015) and suggesting that a complex network of genetic regulation controls telomere length. A detailed functional genetic approach to map the regions of the genome responsible for telomere length variation could identify pathways regulating telomere length.

Overall, the diversity of telomere length distributions observed in these reference strains highlights the plasticity of telomere length regulation and the phenotypic heterogeneity of $C$. reinhardtii reference strains.

\section{Identification of CrTERT encoding the catalytic subunit of telomerase}

Sequence similarity and functional analyses of three independent mutant alleles suggest that the gene model Cre04.g213652 corresponds to, or at least encompasses, the gene encoding the catalytic subunit of telomerase, required to maintain telomere length in $C$. reinhardtii. We propose to rename it CrTERT.

Multiple lines of evidence support this conclusion. First, the predicted protein shares significant sequence similarity with the RNA-binding domain of telomerase from other organisms in its $\mathrm{N}$ terminus. Second, we find a very strong conservation of the C-terminal domain of the proposed CrTERT protein, including two essential aspartates, with the catalytic domain of telomerase not only from plants (maize, A. thaliana, soya, and iris) but also from yeast and human. Third, three independent mutants (tel-m1, tel$\mathrm{m} 2$, and tel-m4) with different insertions of the paromomycin resistance marker in CrTERT, including within its RNA-binding domain (tel-m1) and its catalytic domain (tel-m2) display significantly shorter telomeres than the parental CC4533- strain, which is not the case for other independent mutants from the Clip library located in loci unrelated to telomerase. Finally, telomeres shortened progressively in paromomycin-resistant progenies. However, as we are as of yet unable to detect the mRNA corresponding to CrTERT by either Northern blotting or RT-qPCR (Reverse Transcription Quantitative PCR), possibly because of its low expression, we could not assess CrTERT expression in our study. The identification of additional components of the telomerase holoenzyme and telomere-associated proteins will be the focus of future work.

\section{Telomere shortening, replicative senescence, and alternative maintenance pathways}

After prolonged liquid cultures of multiple independent tel-m1 and tel-m2 mutants, we observed a drastically altered TRF pattern: discrete bands above $1 \mathrm{~kb}$ and a continuous smear of high molecular weight fragments up to the wells. These new TRF signals could correspond to extremely long telomeres, as seen for strain cw15.J14+ and also to DNA molecules with abnormal structures, such as G-quartets, other secondary structures, or single-stranded DNA. These rearrangements might be produced by alternative mechanisms of telomere maintenance or elongation and are reminiscent of telomere profiles observed in type II postsenescent yeast cells (Lundblad \& Blackburn, 1993), ALT cancer cells (Cesare \& Reddel, 2010; Shay et al, 2012), or ALT A. thaliana cell lines (Zellinger et al, 
2007; Akimcheva et al, 2008), in which telomerase-independent recombination mechanisms can lead to very long and heterogeneous telomeres, thus sustaining long-term cell divisions. In these described cases, telomerase is not expressed, telomeres undergo sister-chromatid, and interchromosome homologous recombination using gene conversion, break-induced replication, rolling circle amplification, or yet unknown mechanisms.

Another line of evidence suggesting the occurrence of postsenescence survivors of telomerase-negative cells in C. reinhardtii came from the analysis of the offspring of backcrosses of the tel-m1 and tel-m2 mutants with T222+ reference strain. The CrTERT-mutant progenies experience telomere shortening, and 50\% of them eventually stopped growing after about 6 mo on solid media, a phenotype consistent with replicative senescence. The other $50 \%$ of telomerase-negative progenies have not yet entered senescence at the date of publication of this work (>21 mo). The cells that experienced senescence and generated first generation survivors then showed a complex pattern of moderate growth, followed by cell death and emergence of a new generation of clonal survivors. We do not yet understand the dynamics and variability of the senescence phenotype in these backcrossed haploid progenies. We speculate that for the initial CliP mutants, additional mutations could have been generated that might have acted as suppressors of the senescence phenotype. This would also explain why no growth defect was observed for the initial Clip mutants even after more than $2 \mathrm{yr}$ of maintenance on solid media, whereas senescence, cell death, and postsenescent survivors could be observed after backcrossing these mutants and selecting telomerase-negative haploid progenies. Alternatively, the initial Clip mutants might have already been postsenescence survivors from the beginning. In a future work, it will be interesting to characterize postsenescence survivors by assessing hallmarks of human ALT cancers, including circular extrachromosomal telomeric DNA and up-regulation of telomeric repeat-containing RNA (TERRA) (Cesare \& Reddel, 2010; Arora \& Azzalin, 2015).

Although some fundamental aspects of its telomeres share similarities to other eukaryotes, C. reinhardtii shows a unique combination of telomeric properties that distinguishes it from any other model organism. The characterization of its telomeres at the level of sequence, end structure, length distribution, and maintenance by telomerase or alternative mechanisms provided by this study is an essential step to propose C. reinhardtii as a valuable model organism for telomere biology research.

\section{Materials and Methods}

\section{Strains and growth conditions}

Strains T222+, S24-, CC124-, CC125+, CC503+, CC1009-, CC1010+, and CC4425+ (D66) are described in Gallaher et al (2015). Strains cw15.J3and cw.J14- are cell wall-less strains obtained by crossing. Strains CC620+, CC521+, and CC4350+ are described in the Chlamydomonas Resource Center (https://www.chlamycollection.org/). Strain CC4533is described in the work by Li et al (2016). Strain S1D2 is described in the work by Gross et al (1988), Harris (2001), and Vysotskaia et al (2001). Unless stated otherwise, the cells were grown under continuous illumination either on plates or in agitated 200-ml liquid cultures in TAP medium (Harris, 2009) under low-light, that is, $8 \mu \mathrm{E} \cdot \mathrm{m}^{-2} \cdot \mathrm{s}^{-1}$ or higher light ( $\mathrm{HL}$ ), that is, $80 \mu \mathrm{E} \cdot \mathrm{m}^{-2} \cdot \mathrm{s}^{-1}$. The A. thaliana ecotype Columbia (Col-0) plant was used for the hairpin assay.

\section{gDNA extraction}

Unless stated otherwise, the cells were grown in liquid cultures to early stationary phase $\left(\sim 2 \times 10^{7}\right.$ cells $\left.\cdot \mathrm{mL}^{-1}\right)$ and $150 \mathrm{ml}$ was collected by centrifugation $(5,000 \mathrm{~g}, 5 \mathrm{~min})$. The pellet was frozen at $-80^{\circ} \mathrm{C}$. The cells were then thawed at room temperature and $5 \mathrm{ml}$ of preheated buffer AP1 with RNase (QIAGEN DNA Plant Maxi Kit) was added and cells lysed at $65^{\circ} \mathrm{C}$ for $2 \mathrm{~h}$. After lysis, gDNA was extracted according to the manufacturer's protocol (QIAGEN DNA Plant Maxi Kit). For PETRA and hairpin assays, gDNA was extracted using the CTAB method as described in Borevitz et al (2003).

\section{Telomere PCR and sequencing}

Bulk gDNA was denatured at $95^{\circ} \mathrm{C}$ during 5 min. End labeling reactions (total volume $6 \mu \mathrm{l}$ ) contained $100 \mathrm{ng}$ of bulk gDNA, 1× New England Biolabs restriction buffer 4, dCTP $100 \mu \mathrm{M}$, and 1 unit of terminal transferase (New England Biolabs, NEB) and was carried out at $37^{\circ} \mathrm{C}$ during $30 \mathrm{~min}$, then $65^{\circ} \mathrm{C}$ during $10 \mathrm{~min}$, and $94^{\circ} \mathrm{C}$ during $5 \mathrm{~min}$. The end-labeled telomeres were then amplified with the primers 169M (poly-G-containing primer) and oT1090 targeting the subtelomere/telomere junction common to 10 telomeres of eight chromosomes (Table S1). PCR reactions ( $40 \mu \mathrm{l}$ ) contained the endlabeled DNA, $200 \mu \mathrm{M}$ of dNTPs, primers at $0.5 \mu \mathrm{M}$ for 0 T1090 and 0.75 $\mu \mathrm{M}$ for $169 \mathrm{M}, 1 \times$ Taq Mg-free buffer (NEB), and $2.5 \mathrm{U}$ of standard Taq polymerase (NEB). The PCR conditions were as follows: $94^{\circ} \mathrm{C} 3 \mathrm{~min}$; 32 cycles of $94^{\circ} \mathrm{C} 20 \mathrm{~s}, 60^{\circ} \mathrm{C} 40 \mathrm{~s}$, and $68^{\circ} \mathrm{C} 20 \mathrm{~s}$; and $68^{\circ} \mathrm{C} 5 \mathrm{~min}$.

For sequencing, PCR products were ligated for $1 \mathrm{~h}$ at $16^{\circ} \mathrm{C}$ in a pDrive plasmid. $2 \mu \mathrm{l}$ of the ligation product was transformed into competent bacteria (PCR cloning kit; QIAGEN). Bacteria were plated on LB + ampicillin $(100 \mu \mathrm{g} / \mathrm{ml})+$ IPTG $(50 \mu \mathrm{M})+$ X-gal $(80 \mu \mathrm{g} / \mathrm{ml})$ medium overnight at $37^{\circ} \mathrm{C}$. Plasmids were extracted and purified (Millipore Plasmid Miniprep 96 Kit and Manifold) after $24 \mathrm{~h}$ of culture of white colonies in $1 \mathrm{ml}$ of LB $2 X+$ ampicillin $(100 \mu \mathrm{g} / \mathrm{ml})$ in a 96-well microplate. DNA insertion in plasmids was verified by ECoRI (NEB) digestion. Plasmids were Sanger-sequenced with the M13-PU primer (Eurofins Genomics).

\section{Isolation of nuclei}

Nuclear fraction was prepared from cell wall-mutant CC4350+. Exponentially growing cells ( $2 \mathrm{~d}$ in liquid culture) were gently spun and thoroughly resuspended in $90 \mathrm{ml}$ buffer A per liter of culture (25 $\mathrm{mM}$ Hepes- $\mathrm{NaOH}, \mathrm{pH} 7.5,20 \mathrm{mM} \mathrm{KCl}, 20 \mathrm{mM} \mathrm{MgCl} 2,600 \mathrm{mM}$ sucrose, $10 \%$ glycerol, and $5 \mathrm{mM} \mathrm{DTT}$ ). Triton X-100 was first diluted in $10 \mathrm{ml}$ of buffer A per liter of culture and subsequently added drop wise to the cells while swirling them gently, to a final concentration of $0.5 \%$. Nuclei were pelleted at $800 \mathrm{~g}$ for 2-4 min. Using a paintbrush, the pellet was gently resuspended in fresh buffer A without Triton $\mathrm{X}$-100. After centrifugation, the integrity of nuclei $(1-5 \mu \mathrm{l})$ was checked by fluorescent microscopy using DAPI/vectashield $(5 \mu \mathrm{l})$ 
staining. Nuclei were resuspended in buffer B (2.5\% Ficoll 400, $0.5 \mathrm{M}$ sorbitol, $0.008 \%$ spermidine, $50 \%$ glycerol, and $1 \mathrm{mM}$ DTT), using 1-2 $\mathrm{ml}$ per $200 \mathrm{ml}$ of original culture volume. For storage, nuclei were frozen in liquid nitrogen and stored at $-80^{\circ} \mathrm{C}$.

\section{MNase hypersensitivity assay}

MNase hypersensitivity assay was based on Lodha \& Schroda (2005). $1 \mathrm{ml}$ of nuclei isolated from C. reinhardtii strain CC4350+ cw15 mt+ in buffer B was thawed on ice. To collect nuclei, the sample was spun at maximal speed for $15 \mathrm{~s}$ and resuspended in 500 $\mu \mathrm{l}$ of $1 \times \mathrm{MN}$ buffer (50 mM Tris- $\mathrm{HCl}, \mathrm{pH} 8.0$, and $5 \mathrm{mM} \mathrm{CaCl}$ ). Reactions of total volume of $110 \mu \mathrm{l}$ were carried out in $1 \times \mathrm{MN}$ buffer using $60 \mu \mathrm{l}$ of sample and different amounts of MNase units (Fermentas). gDNA from C. reinhardtii CC4350+ ( 750 ng) was used as a control for enzyme activity and digested with $15 \mathrm{U}$ of nuclease. The samples were incubated 3 min at room temperature, and reactions were stopped by adding $110 \mu \mathrm{l}$ of STOP buffer (1\% SDS and $50 \mathrm{mM}$ EDTA). Proteins were then denatured for $45 \mathrm{~min}$ at $65^{\circ} \mathrm{C}$ and DNA was extracted using $500 \mu \mathrm{l}$ of phenol:chloroform:isoamyl alcohol (25:24:1) using Phase Trap A (Peqlab). Aqueous phase was precipitated by adding $42 \mu \mathrm{l}$ of $3 \mathrm{M} \mathrm{NaOAc}$ and $840 \mu \mathrm{l}$ of $96 \%$ ethanol. DNA was pelleted by centrifugation for $10 \mathrm{~min}$ at maximal speed. The pellet was washed with $70 \%$ ethanol, dried, and resuspended in $25 \mu \mathrm{l} \mathrm{H}_{2} \mathrm{O}$. $6 \times$ loading dye ( $6 \mu \mathrm{l}$ ) was added prior loading onto $1.5 \%$ agarose gel. DNA was stained with ethidium bromide (1\% solution; Applichem), blotted onto uncharged membrane (Amersham), and hybridized with a $\left(\mathrm{T}_{4} \mathrm{AG}_{3}\right)_{3}$ probe. After scanning, the membrane was stripped and reprobed using 18S-derived probe.

\section{PETRA and hairpin assay}

Telomere length of individual chromosomes was determined by Primer Extension Telomere Repeat Amplification (PETRA) as previously described (Watson et al, 2016). For primer extension by the \$29 polymerase, we used the $C$. reinhardtii-specific PETRA-T oligonucleotide 5'-CTCTAGACTGTGAGACTTGGACTACCCTAAAACCCT-3' (Table S1). For specific chromosome arms, we used subtelomeric oligonucleotides 1R: 5'-TACTTGTGTGTGCTGTGCGT-3', 9R: 5'-ACAGCACAATACAGTATATA-3', and 10R: 5'-AACGTCCTCGTGAGACCACC-3' (Table S1). The hairpin assay for detecting blunt-ended telomeres was performed as previously described (Kazda et al, 2012). Southern hybridization was performed with a [ ${ }^{32} \mathrm{P}$ ]ATP-labeled (TTTTAGGG) 3 probe. PETRA membrane was also hybridized with [ ${ }^{32} \mathrm{P}$ ]ATP-labeled 1-kb ladder (Thermo Fisher Scientific).

\section{TRF and in-gel hybridization analyses}

$2 \mu \mathrm{g}$ of gDNA was digested in $300 \mu \mathrm{l}$ with a cocktail of six restriction enzymes (Pstl, BamHI, Mnll, Fokl, Taql, and Mspl; 20 units each). Digestion products were isopropanol precipitated, resuspended in loading buffer (gel loading dye, Purple 6X, New England Biolabs) and resolved on a $1.5 \%$ agarose gel for $4 \mathrm{~h}$ at $150 \mathrm{~V}$. The gel was then soaked in a denaturation bath $(0.4 \mathrm{M} \mathrm{NaOH}$ and $1 \mathrm{M} \mathrm{NaCl})$ for $20 \mathrm{~min}$ and transferred overnight by capillarity to a charged nylon membrane (Hybond XL; GE Healthcare). The CHSB Chlamydomonas telomere-specific oligonucleotide probe (Fulneckova et al, 2013)
(5'-GTtTTAGGGTTTTAGGgTtTTAGGGTTTTAG-3', Table S1) was ${ }^{32} \mathrm{P}$ labeled at the $5^{\prime}$ terminus with ATP $\left(y^{-32} \mathrm{P}\right)$ by the T4 polynucleotide kinase (New England Biolabs). The membrane was hybridized using the Rapid-hyb Buffer protocol (GE Healthcare). In brief, the membrane was prehybridized at $42^{\circ} \mathrm{C}$ in Rapid-hyb buffer for $1 \mathrm{~h}$, then the radioactive probe $(20 \mathrm{pmol})$ was added, and the incubation was continued for $1 \mathrm{~h}$. The membrane was washed consecutively with $5 \times \mathrm{SSC}, 0.5 \% \mathrm{SDS}\left(42^{\circ} \mathrm{C}\right.$ for $\left.10 \mathrm{~min}\right) ; 5 \times \mathrm{SSC}, 0.1 \%$ $\operatorname{SDS}\left(42^{\circ} \mathrm{C}\right.$ for $\left.20 \mathrm{~min}\right)$; and $1 \times \mathrm{SSC}, 0.1 \% \operatorname{SDS}\left(25^{\circ} \mathrm{C}\right.$ for $\left.30 \mathrm{~min}\right)$. A phosphor screen was exposed to the membrane and imaged with a Typhoon FLA 9500 scanner (GE Healthcare). Average telomere length was assessed using Image $1.49 \mathrm{~V}(\mathrm{NIH})$ by measuring the peak of the telomere length distribution signal. For multimodal telomere length profiles, the multiple peaks were measured. We used TeloTool, a software for TRF analysis with a built-in probe intensity correction algorithm (Gohring et al, 2014), to verify that unequal telomeric probe binding was negligible in our conditions. For in-gel hybridization analysis, gDNA was digested by the cocktail of enzymes following the same procedure, with some samples being pretreated with ExOT (50 units for $2 \mathrm{~h}$ at $25^{\circ} \mathrm{C}$; New England Biolabs), which degrades single-stranded 3' DNA extension and generates blunt ends, similarly to Exol. Samples were then run in a 1× Trisborate EDTA (TBE) $0.75 \%$ agarose gel in $1 \times$ TBE buffer for $18 \mathrm{~h}$ at $20 \mathrm{~V}$. The gel was then dried and hybridized overnight at $37^{\circ} \mathrm{C}$ with radioactively labeled probes (oT0958, G-probe and OT0959, C-probe, Table S1) in hybridization buffer ( $5 \times$ SSC, $5 \mu \mathrm{M}$ inorganic pyrophosphate, $1 \mathrm{mM} \mathrm{Na}_{2} \mathrm{HPO}_{4}$, 5× Denhardt's solution, $40 \mathrm{nM} \mathrm{ATP}$, and $20 \mu \mathrm{g} / \mathrm{ml}$ salmon sperm DNA). The gel was then washed three times for $30 \mathrm{~min}$ at room temperature with $0.25 \times$ SSC and imaged as for Southern blots. For loading controls, the gel was then transferred in denaturing conditions on a charged nylon membrane and hybridized again with the same probes.

\section{Supplementary Information}

Supplementary Information is available at https://doi.org/10.26508/lsa. 201900315.

\section{Acknowledgements}

We thank Erin Henninger for her help in setting up the in-gel experiment. We thank the Chlamydomonas Mutant Library Group at Princeton University, the Carnegie Institution for Science, and the Chlamydomonas Resource Center at the University of Minnesota for providing the indexed Chlamydomonas insertional mutants. This work was supported by the Agence Nationale pour la Recherche (ANR) grant “AlgaTelo" (ANR-17-CE20-0002-01) to Z Xu, la Fondation de la Recherche Médicale (MTT “équipe labellisée") and the ANR grant "InTelo" (ANR-16-CE12-0026) to MT Teixeira, the "Initiative d'Excellence" program from the French State (Grant "DYNAMO," ANR-11-LABX0011), and by the Ministry of Education, Youth and Sports of the Czech Republic, European Regional Development Fund-Project "REMAP" (No. CZ.02.1.01/0.0/0.0/15_003/0000479) to K Riha.

\section{Author Contributions}

S Eberhard: conceptualization, formal analysis, supervision, investigation, methodology, and writing-original draft, review, and editing. 
S Valuchova: conceptualization, formal analysis, investigation, methodology, and writing-review and editing.

J Ravat: formal analysis, investigation, and writing-review and editing

J Fulneček: formal analysis, investigation, methodology, and writing-review and editing.

P Jolivet: formal analysis, investigation, methodology, and writing-review and editing.

S Bujaldon: formal analysis, investigation, and writing-review and editing.

SD Lemaire: formal analysis and writing-original draft, review, and editing.

F-A Wollman: conceptualization, formal analysis, and writing-review and editing.

MT Teixeira: conceptualization, formal analysis, and writing-review and editing.

K Riha: conceptualization, formal analysis, supervision, and writing-review and editing.

Z Xu: conceptualization, formal analysis, supervision, investigation, methodology, and writing-original draft, review, and editing.

\section{Conflict of Interest Statement}

The authors declare that they have no conflict of interest.

\section{References}

Akimcheva S, Zellinger B, Riha K (2008) Genome stability in Arabidopsis cells exhibiting alternative lengthening of telomeres. Cytogenet Genome Res 122: 388-395. doi:10.1159/000167827

Aksenova AY, Han G, Shishkin AA, Volkov KV, Mirkin SM (2015) Expansion of interstitial telomeric sequences in yeast. Cell Rep 13: 1545-1551. doi:10.1016/j.celrep.2015.10.023

Arneric M, Lingner J (2007) Tel1 kinase and subtelomere-bound Tbf1 mediate preferential elongation of short telomeres by telomerase in yeast. EMBO Rep 8: 1080-1085. doi:10.1038/sj.embor.7401082

Arora R, Azzalin CM (2015) Telomere elongation chooses TERRA ALTernatives. RNA Biol 12: 938-941. doi:10.1080/15476286.2015.1065374

Askree SH, Yehuda T, Smolikov S, Gurevich R, Hawk J, Coker C, Krauskopf A, Kupiec M, McEachern MJ (2004) A genome-wide screen for Saccharomyces cerevisiae deletion mutants that affect telomere length. Proc Natl Acad Sci USA 101: 8658-8663. doi:10.1073/ pnas. 0401263101

Azzalin CM, Nergadze SG, Giulotto E (2001) Human intrachromosomal telomeric-like repeats: Sequence organization and mechanisms of origin. Chromosoma 110: 75-82. doi:10.1007/s004120100135

Borevitz JO, Liang D, Plouffe D, Chang HS, Zhu T, Weigel D, Berry CC, Winzeler E, Chory J (2003) Large-scale identification of single-feature polymorphisms in complex genomes. Genome Res 13: 513-523. doi:10.1101/gr.541303

Burr B, Burr FA, Matz EC, Romero-Severson J (1992) Pinning down loose ends: Mapping telomeres and factors affecting their length. Plant cell 4: 953-960. doi:10.2307/3869462

Cesare AJ, Reddel RR (2010) Alternative lengthening of telomeres: Models, mechanisms and implications. Nat Rev Genet 11: 319-330. doi:10.1038/ nrg2763

Cetin B, Cleveland DW (2010) How to survive aneuploidy. Cell 143: 27-29. doi:10.1016/j.cell.2010.09.030
Chang HY, Lawless C, Addinall SG, Oexle S, Taschuk M, Wipat A, Wilkinson DJ, Lydall D (2011) Genome-wide analysis to identify pathways affecting telomere-initiated senescence in budding yeast. G3 (Bethesda) 1: 197-208. doi:10.1534/g3.111.000216

Clark DJ (2010) Nucleosome positioning, nucleosome spacing and the nucleosome code. J Biomol Struct Dyn 27: 781-793. doi:10.1080/ 073911010010524945

Cross FR, Umen JG (2015) The Chlamydomonas cell cycle. Plant J 82: 370-392. doi:10.1111/tpj.12795

Epel ES, Blackburn EH, Lin J, Dhabhar FS, Adler NE, Morrow JD, Cawthon RM (2004) Accelerated telomere shortening in response to life stress. Proc Natl Acad Sci USA 101: 17312-17315. doi:10.1073/pnas.0407162101

Fajkus J, Sykorova E, Leitch AR (2005) Techniques in plant telomere biology. BioTechniques 38: 233-243. doi:10.2144/05382rv01

Findinier J, Delevoye C, Cohen MM (2019) The dynamin-like protein Fzl promotes thylakoid fusion and resistance to light stress in Chlamydomonas reinhardtii. PLoS Genet 15: e1008047. doi:10.1371/ journal.pgen.1008047

Forstemann K, Hoss M, Lingner J (2000) Telomerase-dependent repeat divergence at the 3 ' ends of yeast telomeres. Nucleic Acids Res 28: 2690-2694. doi:10.1093/nar/28.14.2690

Fulcher N, Derboven E, Valuchova S, Riha K (2014) If the cap fits, wear it: An overview of telomeric structures over evolution. Cell Mol Life Sci 71: 847-865. doi:10.1007/s00018-013-1469-z

Fulcher N, Teubenbacher A, Kerdaffrec E, Farlow A, Nordborg M, Riha K (2015) Genetic architecture of natural variation of telomere length in Arabidopsis thaliana. Genetics 199: 625-635. doi:10.1534/ genetics.114.172163

Fulnečková J, Hasíková T, Fajkus J, Lukešová A, Eliáš M, Sýkorová E (2012) Dynamic evolution of telomeric sequences in the green algal order Chlamydomonadales. Genome Biol Evol 4: 248-264. doi:10.1093/gbe/ evs007

Fulnecková J, Sevcíková T, Fajkus J, Lukesová A, Lukes M, Vlcek C, Lang BF, Kim E, Elias M, Sykorová E (2013) A broad phylogenetic survey unveils the diversity and evolution of telomeres in eukaryotes. Genome Biol Evol 5: 468-483. doi:10.1093/gbe/evt019

Fulnečková J, Ševčíková T, Lukešová A, Sýkorová E (2015) Transitions between the Arabidopsis-type and the human-type telomere sequence in green algae (clade Caudivolvoxa, Chlamydomonadales). Chromosoma 125: 437-451. doi:10.1007/s00412-015-0557-2

Gallaher SD, Fitz-Gibbon ST, Glaesener AG, Pellegrini M, Merchant SS (2015) Chlamydomonas genome resource for laboratory strains reveals a mosaic of sequence variation, identifies true strain histories, and enables strain-specific studies. Plant Cell 27: 2335-2352. doi:10.1105/ tpc.15.00508

Gaspin C, Rami JF, Lescure B (2010) Distribution of short interstitial telomere motifs in two plant genomes: Putative origin and function. BMC Plant Biol 10: 283. doi:10.1186/1471-2229-10-283

Gatbonton T, Imbesi M, Nelson M, Akey JM, Ruderfer DM, Kruglyak L, Simon JA, Bedalov A (2006) Telomere length as a quantitative trait: Genomewide survey and genetic mapping of telomere length-control genes in yeast. PLoS Genet 2: e35. doi:10.1371/journal.pgen.0020104

Giraud-Panis MJ, Teixeira MT, Geli V, Gilson E (2010) CST meets shelterin to keep telomeres in check. Mol Cell 39: 665-676. doi:10.1016 j.molcel.2010.08.024

Gohring J, Fulcher N, Jacak J, Riha K (2014) TeloTool: A new tool for telomere length measurement from terminal restriction fragment analysis with improved probe intensity correction. Nucleic Acids Res 42: e21. doi:10.1093/nar/gkt1315

Greenwood J, Patel H, Cech TR, Cooper JP (2018) Fission yeast telosomes: Noncanonical histone-containing chromatin structures dependent on 
shelterin and RNA. Nucleic Acids Res 46: 8865-8875. doi:10.1093/nar/ gky605

Gross CH, Ranum LP, Lefebvre PA (1988) Extensive restriction fragment length polymorphisms in a new isolate of Chlamydomonas reinhardtii. Curr Genet 13: 503-508. doi:10.1007/bf02427756

Hails T, Huttner O, Day A (1995) Isolation of a Chlamydomonas reinhardtii telomere by functional complementation in yeast. Curr Genet 28 : 437-440. doi:10.1007/bf00310812

Harley CB, Futcher AB, Greider CW (1990) Telomeres shorten during ageing of human fibroblasts. Nature 345: 458-460. doi:10.1038/345458a0

Harris EH (2001) Chlamydomonas as a model organism. Annu Rev Plant Physiol Plant Mol Biol 52: 363-406. doi:10.1146/ annurev.arplant.52.1.363

Harris EH (2009) The Chlamydomonas Sourcebook (Second Edition). Canada: Elsevier Academic Press.

Heacock M, Spangler E, Riha K, Puizina J, Shippen DE (2004) Molecular analysis of telomere fusions in Arabidopsis: Multiple pathways for chromosome end-joining. EMBO J 23: 2304-2313. doi:10.1038/ sj.emboj.7600236

Johnston SD, Lew JE, Berman J (1999) Gbp1p: A protein with RNA recognition motifs, binds single-stranded telomeric DNA and changes its binding specificity upon dimerization. Mol Cell Biol 19: 923-933. doi:10.1128/ mcb.19.1.923

Kazda A, Zellinger B, Rossler M, Derboven E, Kusenda B, Riha K (2012) Chromosome end protection by blunt-ended telomeres. Genes Dev 26: 1703-1713. doi:10.1101/gad.194944.112

Lewis KA, Wuttke DS (2012) Telomerase and telomere-associated proteins: Structural insights into mechanism and evolution. Structure 20: 28-39. doi:10.1016/j.str.2011.10.017

Li X, Zhang R, Patena W, Gang SS, Blum SR, Ivanova N, Yue R, Robertson JM, Lefebvre P, Fitz-Gibbon ST, et al (2016) An indexed, mapped mutant library enables reverse genetics studies of biological processes in Chlamydomonas reinhardtii. Plant Cell 28: 367-387. doi:10.1105/ tpc.15.00465

Lingner J, Cech TR, Hughes TR, Lundblad V (1997a) Three Ever Shorter Telomere (EST) genes are dispensable for in vitro yeast telomerase activity. Proc Natl Acad Sci USA 94: 11190-11195. doi:10.1073/ pnas.94.21.11190

Lingner J, Hughes TR, Shevchenko A, Mann M, Lundblad V, Cech TR (1997b) Reverse transcriptase motifs in the catalytic subunit of telomerase. Science 276: 561-567. doi:10.1126/science.276.5312.561

Liti G, Haricharan S, Cubillos FA, Tierney AL, Sharp S, Bertuch AA, Parts L, Bailes E, Louis EJ (2009) Segregating YKU80 and TLC1 alleles underlying natural variation in telomere properties in wild yeast. PLoS Genet 5: e1000659. doi:10.1371/journal.pgen.1000659

Lodha M, Schroda M (2005) Analysis of chromatin structure in the control regions of the chlamydomonas HSP70A and RBCS2 genes. Plant Mol Biol 59: 501-513. doi:10.1007/s11103-005-0450-0

Lundblad V, Blackburn EH (1993) An alternative pathway for yeast telomere maintenance rescues est1- senescence. Cell 73: 347-360. doi:10.1016/ 0092-8674(93)90234-h

Lundblad V, Szostak JW (1989) A mutant with a defect in telomere elongation leads to senescence in yeast. Cell 57: 633-643. doi:10.1016/00928674(89)90132-3

Marzec P, Armenise C, Perot G, Roumelioti FM, Basyuk E, Gagos S, Chibon F, Dejardin J (2015) Nuclear-receptor-mediated telomere insertion leads to genome instability in ALT cancers. Cell 160: 913-927. doi:10.1016/ j.cell.2015.01.044

Merchant SS, Prochnik SE, Vallon O, Harris EH, Karpowicz SJ, Witman GB, Terry A, Salamov A, Fritz-Laylin LK, Marechal-Drouard L, et al (2007) The Chlamydomonas genome reveals the evolution of key animal and plant functions. Science 318: 245-250. doi:10.1126/ science. 1143609

Meyne J, Baker RJ, Hobart HH, Hsu TC, Ryder OA, Ward OG, Wiley JE, WursterHill DH, Yates TL, Moyzis RK (1990) Distribution of non-telomeric sites of the (TTAGGG)n telomeric sequence in vertebrate chromosomes. Chromosoma 99: 3-10. doi:10.1007/bf01737283

Millet C, Ausiannikava D, Le Bihan T, Granneman S, Makovets S (2015) Cell populations can use aneuploidy to survive telomerase insufficiency. Nat Commun 6: 8664. doi:10.1038/ncomms9664

Millet C, Makovets S (2016) Aneuploidy as a mechanism of adaptation to telomerase insufficiency. Curr Genet 62: 557-564. doi:10.1007/s00294015-0559-x

Nakamura TM, Morin GB, Chapman KB, Weinrich SL, Andrews WH, Lingner J, Harley CB, Cech TR (1997) Telomerase catalytic subunit homologs from fission yeast and human. Science 277: 955-959. doi:10.1126/ science.277.5328.955

Oguchi K, Liu H, Tamura K, Takahashi H (1999) Molecular cloning and characterization of AtTERT, a telomerase reverse transcriptase homolog in Arabidopsis thaliana. FEBS Lett 457: 465-469. doi:10.1016/ s0014-5793(99)01083-2

Palm W, de Lange T (2008) How shelterin protects mammalian telomeres. Annu Rev Genet 42: 301-334. doi:10.1146/ annurev.genet.41.110306.130350

Petracek ME, Berman J (1992) Chlamydomonas reinhardtii telomere repeats form unstable structures involving guanine-guanine base pairs. Nucleic Acids Res 20: 89-95. doi:10.1093/nar/20.189

Petracek ME, Konkel LMC, Kable ML, Berman J (1994) A Chlamydomonas protein that binds single-stranded G-strand telomere DNA. EMBO J 13: 3648-3658. doi:10.1002/j.1460-2075.1994.tb06672.x

Petracek ME, Lefebvre PA, Silflow CD, Berman J (1990) Chlamydomonas telomere sequences are A+T-rich but contain three consecutive G-C basepairs. Proc Natl Acad Sci USA 87: 8222-8226. doi:10.1073/ pnas.87.21.8222

Pfeiffer V, Lingner J (2013) Replication of telomeres and the regulation of telomerase. Cold Spring Harbor Perspect Biol 5: a010405. doi:10.1101/ cshperspect.a010405

Platt JM, Ryvkin P, Wanat JJ, Donahue G, Ricketts MD, Barrett SP, Waters HJ, Song S, Chavez A, Abdallah KO, et al (2013) Rap1 relocalization contributes to the chromatin-mediated gene expression profile and pace of cell senescence. Genes Dev 27: 1406-1420. doi:10.1101/ gad. 218776.113

Raices M, Maruyama H, Dillin A, Karlseder J (2005) Uncoupling of longevity and telomere length in C. elegans. PLoS Genet 1: e30. doi:10.1371/ journal.pgen.0010081

Rochaix JD (1995) Chlamydomonas reinhardtii as the photosynthetic yeast Annu Rev Genet 29: 209-230. doi:10.1146/annurev.ge.29.120195.001233

Romano GH, Harari Y, Yehuda T, Podhorzer A, Rubinstein L, Shamir R, Gottlieb A, Silberberg Y, Pe'er D, Ruppin E, et al (2013) Environmental stresses disrupt telomere length homeostasis. PLoS Genet 9: e1003721. doi:10.1371/journal.pgen.1003721

Sasso S, Stibor H, Mittag M, Grossman AR (2018) From molecular manipulation of domesticated Chlamydomonas reinhardtii to survival in nature. Elife 7: e39233.

Scaife MA, Nguyen GT, Rico J, Lambert D, Helliwell KE, Smith AG (2015) Establishing Chlamydomonas reinhardtii as an industrial biotechnology host. Plant / 82: 532-546. doi:10.1111/tpj.12781

Scranton MA, Ostrand JT, Fields FJ, Mayfield SP (2015) Chlamydomonas as a model for biofuels and bio-products production. Plant J 82: 523-531. doi:10.1111/tpj.12780

Shakirov EV, Shippen DE (2004) Length regulation and dynamics of individual telomere tracts in wild-type Arabidopsis. Plant Cell 16: 1959-1967. doi:10.1105/tpc.104.023093 
Shay JW, Reddel RR, Wright WE (2012) Cancer. Cancer and telomeres: An ALTernative to telomerase. Science 336: 1388-1390. doi:10.1126/ science. 1222394

Uchida W, Matsunaga S, Sugiyama R, Kawano S (2002) Interstitial telomerelike repeats in the Arabidopsis thaliana genome. Genes Genet Syst 77: 63-67. doi:10.1266/ggs.77.63

Ungar L, Yosef N, Sela Y, Sharan R, Ruppin E, Kupiec M (2009) A genome-wide screen for essential yeast genes that affect telomere length maintenance. Nucleic Acids Res 37: 3840-3849. doi:10.1093/nar/ gkp259

von Zglinicki T (2000) Role of oxidative stress in telomere length regulation and replicative senescence. Ann New York Acad Sci 908: 99-110. doi:10.1111/j.1749-6632.2000.tb06639.x

Vysotskaia VS, Curtis DE, Voinov AV, Kathir P, Silflow CD, Lefebvre PA (2001) Development and characterization of genome-wide single nucleotide polymorphism markers in the green alga Chlamydomonas reinhardtii. Plant Physiol 127: 386-389. doi:10.1104/pp.010485

Walmsley RM, Petes TD (1985) Genetic control of chromosome length in yeast. Proc Natl Acad Sci USA 82: 506-510. doi:10.1073/pnas.82.2.506

Watson JM, Platzer A, Kazda A, Akimcheva S, Valuchova S, Nizhynska V, Nordborg M, Riha K (2016) Germline replications and somatic mutation accumulation are independent of vegetative life span in Arabidopsis. Proc Natl Acad Sci USA 113: 12226-12231. doi:10.1073/ pnas. 1609686113
Wellinger RJ, Zakian VA (2012) Everything you ever wanted to know about Saccharomyces cerevisiae telomeres: Beginning to end. Genetics 191: 1073-1105. doi:10.1534/genetics.111.137851

Wright JH, Gottschling DE, Zakian VA (1992) Saccharomyces telomeres assume a non-nucleosomal chromatin structure. Genes Dev 6: 197-210. doi:10.1101/gad.6.2.197

Wu RA, Upton HE, Vogan JM, Collins K (2017) Telomerase mechanism of telomere synthesis. Annu Rev Biochem 86: 439-460. doi:10.1146/ annurev-biochem-061516-045019

Zakian VA (1995) Telomeres: Beginning to understand the end. Science 270: 1601-1607. doi:10.1126/science.270.5242.1601

Zellinger B, Akimcheva S, Puizina J, Schirato M, Riha K (2007) Ku suppresses formation of telomeric circles and alternative telomere lengthening in Arabidopsis. Mol Cell 27: 163-169. doi:10.1016/j.molcel.2007.05.025

Zhu L, Hathcock KS, Hande P, Lansdorp PM, Seldin MF, Hodes RJ (1998) Telomere length regulation in mice is linked to a novel chromosome locus. Proc Natl Acad Sci USA 95: 8648-8653. doi:10.1073/ pnas.95.15.8648

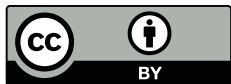

License: This article is available under a Creative Commons License (Attribution 4.0 International, as described at https://creativecommons.org/ licenses/by/4.0/). 